\title{
Aus Hochschulen und Institutionen / Academic and Educational News
}

\section{Neubesetzung an der Spitze des BKG}

\section{Prof. Dr. Paul Becker ist Präsident des Amtes}

Das Bundesamt für Kartographie und Geodäsie (BKG) hat einen neuen Präsidenten. Prof. Dr. Paul Becker, zuletzt Vizepräsident beim Deutschen Wetterdienst (DWD) in Offenbach, hat am 1. April 2019 das Amt an der Spitze des BKG angetreten.

Der studierte Meteorologe arbeitet seit vielen Jahren eng mit dem BKG im Rahmen der Group on Earth Observations zusammen. Ziel ist es, die globalen Erdbeobachtungsinfrastrukturen besser zu koordinieren und die gewonnenen Daten leichter verfügbar zu machen. Neben seiner beruflichen Tätigkeit als Vizepräsident des DWD hat Becker eine Honorarprofessur an der Goethe-Universität in Frankfurt am Main und einen Lehrauftrag an der Universität Hamburg inne. Becker: „Ich freue mich auf das neue Amt. Viele der mit der neuen Position verbundenen Herausforderungen knüpfen unmittelbar an die Themen an, die ich in der Vergangenheit bearbeitet habe.“

Eines der wichtigsten Ziele in den nächsten Monaten wird der Aufbau einer elektronischen Plattform sein, die es erlaubt, Informationen der verschiedensten deutschen Behörden zu verknüpfen, um so u. a. die Bearbeitung komplexer interdisziplinärer Fragestellungen, z.B. in der Katastrophenvorsorge, zu unterstützen. Basierend auf der Darstellung von hoch aufgelösten 3D-Modellen, verknüpft mit Niederschlagsklimatologien und Niederschlagsabflussmodellen, könnte z.B. die Entwicklung neuer Hochwasserschutzmaßnahmen gegen sehr kleinräumige, aber intensive Niederschlagsereignisse unterstützt werden. Darüber hinaus soll ein neues Zentrum geschaffen werden, um im Katastrophenfall möglichst rasch genaue Luft- und Satellitenlagebilder vom Ort des Geschehens liefern zu können.

Der neue BKG-Präsident sieht noch weitere wichtige Handlungsfelder, beispielsweise bei der Satellitennavigation: „Die Auswertung der schnell ansteigenden Zahl von neuen Navigationssatelliten wollen wir intensivieren, um letztendlich eine noch genauere Navigation zu ermöglichen und damit Beiträge zum autonomen Fahren und zum Precision Farming zu leisten." Viele der neuen Tätigkeiten

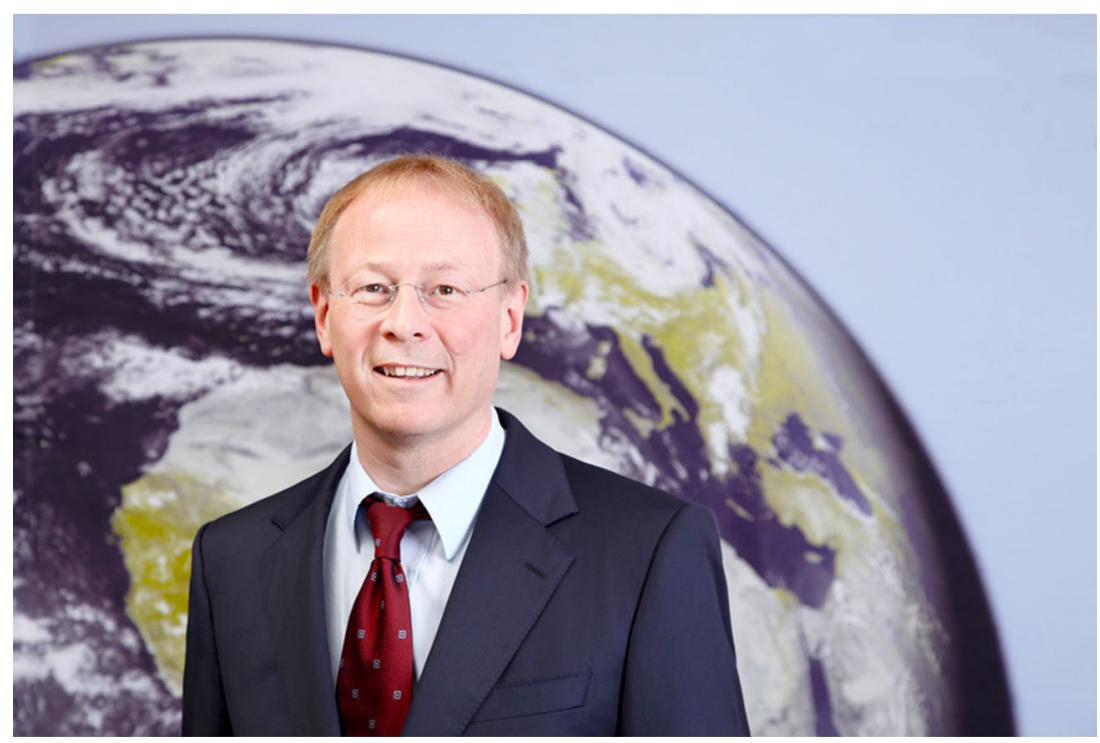

Prof. Dr. Paul Becker 
erfordern eine intensive nationale und internationale Vernetzung. Diese wird daher ein zentraler Aspekt seiner zukünftigen Arbeit sein.

Hintergrundinformationen zum Bundesamt für Kartographie und Geodäsie (BKG):

Als zentraler Dienstleister des Bundes und Kompetenzzentrum für Geoinformation und geodätische Referenzsysteme befasst sich das BKG mit der Beobachtung über die Datenhaltung bis hin zur Analyse, Kombination und Bereitstellung von Geodaten. Von der Arbeit des BKG, einer Behörde im Geschäftsbereich des Bundesministeriums des Innern, für Bau und Heimat (BMI), profitieren insbesondere Bundeseinrichtungen, die öffentliche Verwaltung, Wirtschaft, Wissenschaft - und fast jeder Bürger in Deutschland. Experten aus den verschiedensten Bereichen wie Verkehr, Katastrophenvorsorge, Innere Sicherheit, Energie und Umwelt verwenden Geodaten, Landkarten, Referenzsysteme und Informationsdienste des BKG für ihre Pläne und Untersuchungen. Das BKG unterhält ein Dienstleistungszentrum in Leipzig (www.geodatenzentrum.de) sowie geodätische Observatorien im Inund Ausland.

(Pressemitteilung,presse@bkg.bund.de)

\section{IRO-Landkarte gesucht}

\section{Das IfL bittet um Ihre Mithilfe}

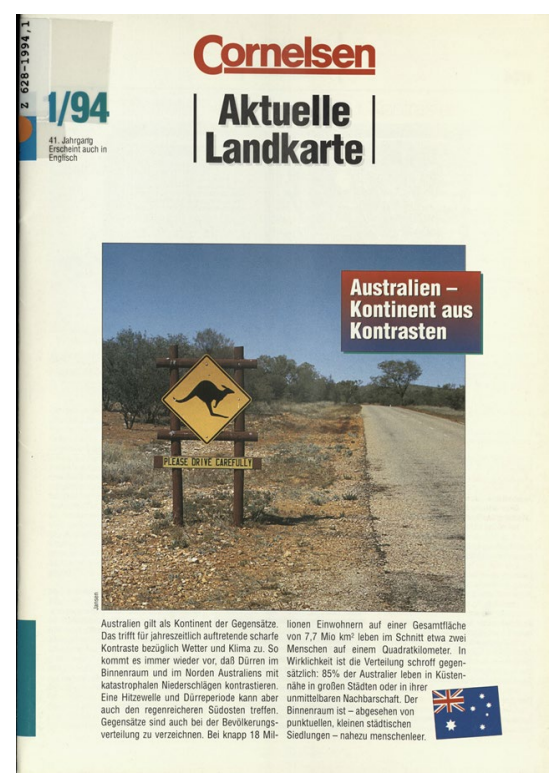

Das Leibniz-Institut für Länderkunde sucht die zwischen 1954 und 1989 erschienene Zeitschrift „Die Aktuelle JRO-Landkarte" sowie die Nachfolger-Zeitschrift „Die aktuelle Landkarte", die im Cornlesen-Verlag bis 2002 erschien. Wer größere Bestände der Zeitschrift besitzt und diese abgeben möchte, wird gebeten, sich mit dem Leiter der Bibliothek im IfL in Verbindung zu setzen:

Dr. Heinz Peter Brogiato

Tel. +49 341600 55-126

h_brogiato@ifl-leipzig.de
Fortsetzung des

Fachinformationsdienstes

Kartographie und

Geobasisdaten

\section{Rückblick und Ausblick}

Der Fachinformationsdienst Kartographie und Geobasisdaten (FID Karten $)^{1}$ geht in die zweite Phase. Der Antrag zur Fortsetzung der vor drei Jahren begonnenen Arbeiten wurde von den Gutachtern der Deutschen Forschungsgemeinschaft (DFG) positiv bewertet und wird nun weitere drei Jahre gefördert. Der FID Karten basiert auf den ehemaligen Sondersammelgebieten 14.1 (Kartographische Veröffentlichungen) und 28.1 (Topographische Karten), deren Schwerpunkte in der Erwerbung sowie in der allgemeinen (Stabi-Kat) und in der spezifischen Erschließung (Fachliteratur in der Bibliographia Cartographica; topographische Karten in der Topo-Liste) $)^{2}$ lagen. Die DFG als Förderer änderte 2014 das bisherige System der überregionalen Literaturversorgung zugunsten des Aufbaus von Fachinformationssystemen, worum sich auch die Kartenabteilung der Staatsbibliothek zu Berlin 2015 erfolgreich beworben hatte. Aus der langjährigen Erfahrung der Nutzerbedarfe und -gewohnheiten wurden für den

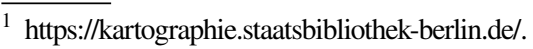

2 StaBiKat: http://stabikat.de/; BC: http:// bc.staatsbibliothek-berlin.de/; Topo-Liste: sbb.berlin/3rl8h6.
}

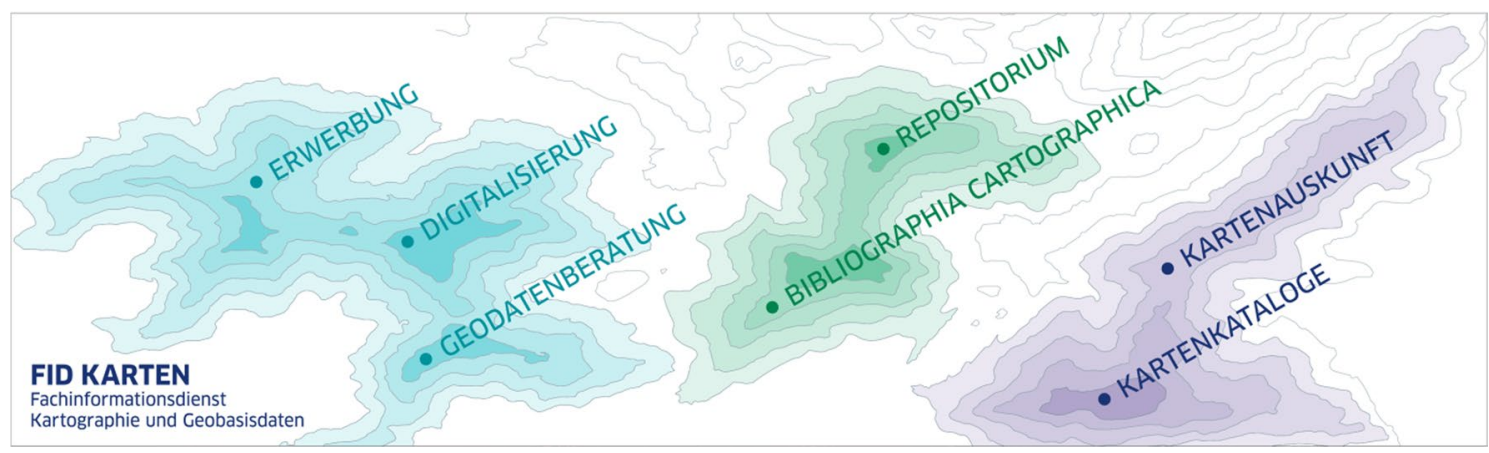


Erstantrag des FID Karten vier Ziele formuliert, die einerseits die bisherigen Angebote schärfen oder erweitern und die andererseits den Aufbau des neuen Service der Geodatenberatung und -vermittlung als Offerte für neue Nutzergruppen mit experimentellem Charakter testen sollten. ${ }^{3}$ Diese Ziele seien zur Erinnerung nochmals aufgeführt:

1. Umbau der Bibliographia Cartographica (BC) zu einer open access Plattform und der Anbindung eines Fachrepositoriums

2. Neuausrichtung der Erwerbungsrichtlinien für kartographische Werke

3. Unterstützung von Projekten und Forschungsvorhaben durch Bereitstellung von Digitalisaten

4. Aufbau einer Vermittlungsstelle zur Beschaffung von Geobasisdaten

Die bereits während der SSG-Förderung bestehende enge Kooperation mit den an der Staats- und Universitätsbibliothek Göttingen installierten SSGs zu Geographie und Thematischen Karten wurden dabei inhaltlich auf den dort neu eingerichteten Fachinformationsdienst Geowissenschaften der festen Erde (FID GEO) abgestimmt. In den Bereichen Erwerbung, Digitalisierung von Karten und des Datenaustausches wurden entsprechend der neuen Aufgabenstellungen Absprachen getroffen, um unnötige Redundanzen zu vermeiden bzw. über den Austausch zu einer schnellen Lösung für die jeweilige Fachcommunity zu kommen. Zudem profitieren die beteiligten Einrichtungen gegenseitig von Entwicklungen, Erfahrungen und Angeboten, so dass inzwischen weitere Kooperationen und Absprachen mit neuen FIDs vorgenommen werden konnten. Genannt seien an dieser Stelle der Fachinformationsdienst Mobilitätsund Verkehrsforschung (FID Move)

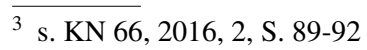

oder regionale FIDs wie zu Ostasien oder Lateinamerika. ${ }^{4}$

Ein Beirat, bestehend aus 7 Mitgliedern, begleitete den Aufbau des FID Karten von Anfang an und konnte so einerseits die Verbindung zur Fachcommunity stärken und andererseits mit dezenten, aber klugen Ratschlägen steuernd eingreifen. Die inhaltliche Zusammensetzung dieses Gremiums aus universitären wie außeruniversitären Wissenschaftlerinnen und Wissenschaftlern vertrat verschiedene Aspekte der Kartographie, Geoinformation, des Geodatenmanagements oder der Geschichte der Kartographie.

Während die Erwerbung von Karten zumeist proaktiv im Rahmen der sonstigen routinemäßigen Einkaufsaktivitäten der Kartenabteilung eingebettet und die Digitalisierung ${ }^{5}$ ebenfalls in das bereits bestehende Angebot integriert werden konnten, waren mit den Aufgabenfeldern im Rahmen des Umbaus der Bibliographie und des Geodatenmanagements neue Tätigkeiten aufzunehmen und entsprechende Strukturen zu schaffen. Insbesondere die Ergänzung der Bibliographie um retrospektiv digitalisierte Publikationen aus kartographischen Fachzeitschriften hat zu einem signifikanten Anstieg ihrer Nutzung geführt. Allein 2018 waren über 18.000 Aktionen in der online frei zugänglichen Datenbank zu verzeichnen. Der Schwerpunkt der Zugriffe erfolgte aus Deutschland, doch sind auch Aktionen aus Polen, den USA, Australien und selbst China zu verzeichnen gewesen (Quelle: Matomo-web analytics).

Die Verlinkung elektronischer Publikationen direkt aus der Recherche heraus wurde zunehmend als ein bequemer Service angenommen, so dass die rückwirkende Einbindung weiterer elektronischer Aufsätze fortgesetzt

${ }^{4}$ FID GEO: https://www.fidgeo.de/; FID MOVE: https://projects.tib.eu/fid-move/startseite/; CrossAsia: https://crossasia.org/; FID Lateinamerika, Karibik und Latino Studies: https://www.iai.spk-berlin.de/bibliothek/fachinformationsdienst.html. wird. Derzeit sind etwa 8.500 elektronische Titel in der Datenbank verknüpft, die Gesamtzahl der enthaltenen Titel beträgt in etwa 56.000. Mit dem Aufbau des Fachrepositoriums wird hingegen eine Verbindung zur Bibliographie erstellt, da sie dieselbe technische Plattform nutzen werden. Diese Verbindung wird künftig unter dem Label CarLi+ (Cartographic Literature plus Repository) geführt und soll damit den doch antiquierten Begriff „Bibliographia“ ablösen und die Internationalität der Fachliteratur dieser Datenbank besser darstellen.

Neben der Fachliteratur beeindrucken auch die Neuzugänge an Karten und Atlanten in gedruckter oder elektronischer Form mit entsprechenden Zahlen. So konnten während der ersten Förderphase, aber auch Dank der Zuwendung der Bundesregierung durch die Beauftragte für Kunst und Medien, insbesondere aktuelle topographische Kartenwerke beispielweise von Afghanistan (TK 50), Armenien (TK 50), Bosnien (TK 25), Bulgarien (TK 50), Georgien (TK 50), Israel (TK 25), Jemen (TK 200), Mali (TK 200), Mazedonien (TK 25, TK 50 und TK 100), Mexiko (TK 50), Slowenien (TK 50, Togo (TK 50) oder Ungarn (TK 25) jeweils komplett angeschafft werden. Darüber hinaus konnten alle je erschienenen gedruckten topographischen Kartenblätter von Mexiko, Spanien und den USA in digitalisierter Form angekauft werden, weitere Ankäufe dieser Art sind bereits in Verhandlung.

Der Service der Beratungs- und Vermittlungsstelle für Geodaten hat mit seiner 2017 durchgeführten Online-Umfrage einen ersten Überblick über die Bedarfe der Fachcommunity gewonnen. ${ }^{6}$ Mit dem im März 2019

\footnotetext{
${ }^{5}$ An dieser Stelle sei der Beuth Hochschule für Technik Berlin, Fachbereich III Bauingenieur- und Geoinformationswesen sehr herzlich für die Kooperation und tatkräftige Unterstuitzung durch Abordnung von studentischen Hilfskräften gedankt.

${ }^{6}$ s. KN 68, 2018, 4, S. 208-210
} 
durchzuführenden Workshop „Bedarfsorientierte Forschungsumgebung: Geodatenberatung und -vermittlung in der Kartenabteilung der Staatsbibliothek ${ }^{\text {*7 }}$ wird das Portfolio weiter geschärft werden können, um die aktuellen Bedarfe der Wissenschaftlerinnen und Wissenschaftler intensiver zu unterstützen. In diesem Zusammenhang wurden als ein weiteres Serviceangebot in der oben bereits genannten Datenbank TopoListe Links zu nationalen Geoportale aufgenommen und zudem Hinweise über Grids \& Datums ${ }^{8}$ ergänzt, um nützliche Informationen über die geodätischen Grundlagen der jeweiligen Landesaufnahmen schnell nachlesen zu können (Abb. 1). Die Einbindung dieser Informationen für die europäischen Länder ist erfolgt, weitere Ergänzungen werden sukzessive fortgesetzt.

Zur Nutzung spezieller Services des FID Karten ist ein Online-Registrierungsverfahren verfügbar. Über die Homepage des FID Karten werden unter dem Reiter „SERVICE“ Formulare für eine zu beantragende Unterstützung für Kartenerwerbungen, thematisch-fokussierte Digitalisierungswünsche oder Geodatenvermittlung bereitgestellt. So ist eine bedarfsorientierte und schnelle Zuordnung gegeben, die uns gleichzeitig einen Überblick über die begleiteten Verfahren gibt und somit ein den Statuten entsprechender und planbarer Umgang mit den verfügbaren Ressourcen sicherstellt.

Noch sind nicht alle Arbeiten, die im Rahmen der ersten Förderphase in Angriff genommen wurden, abgeschlossen. Dennoch kam seitens der DFG die Aufforderung zur Beantragung einer zweiten Förderphase im Turnus der Einreichungsfristen, so dass im Frühjahr 2018 ein

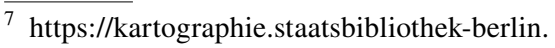
de/fid-karten/.

${ }^{8}$ Es handelt sich dabei um Informationen der American Society for Photogrammetry and Remote Sensing, die in der Zeitschrift Photogrammetric Engineering \& Remote Sensing publiziert werden.
}

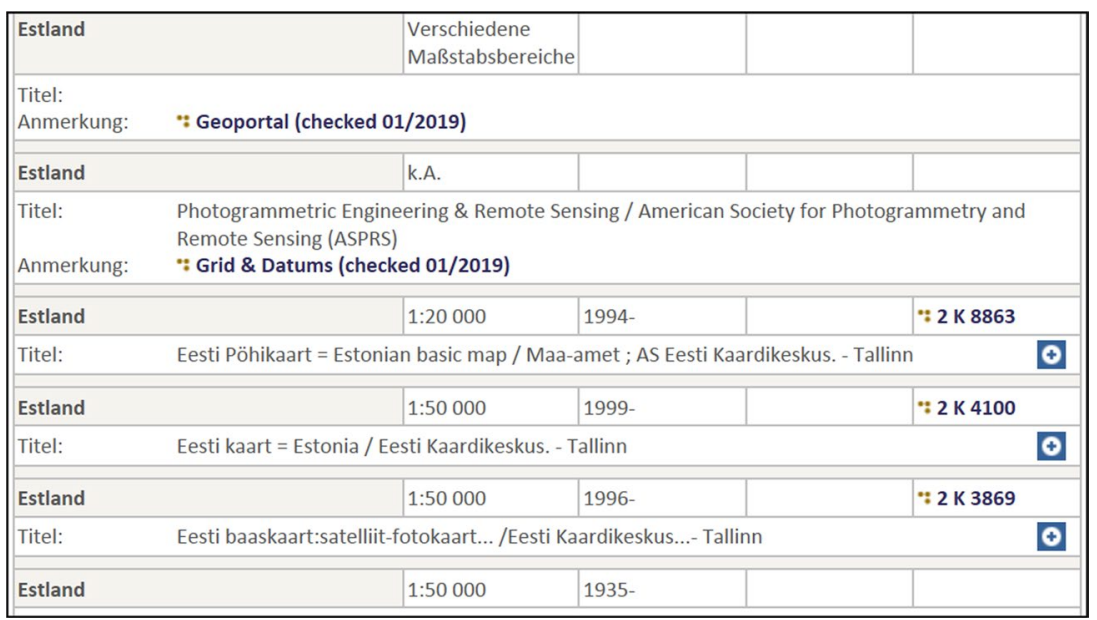

Topo-Liste der Kartenabteilung der Staatsbibliothek zu Berlin (Ausschnitt Beispiel Estland mit Geoportal- und Grid \& Datums-Verlinkungen) sbb.berlin/3rl8h6

Fortsetzungsantrag eingereicht und im Sommer vor dem Gutachtergremium erfolgreich verteidigt wurde. Da das anschließende dreijährige Förderprogramm auch eine stetige Anpassung an aktuelle Bedarfe der Fachcommunities impliziert, können nicht nur laufende Services einfach fortgesetzt werden, sondern es sind neue Ziele oder Projekte zu ermitteln und dafür entsprechende Programme zu formen. Für die zweite Förderphase FID Karten werden 5 Ziele formuliert:

1. Fortsetzung der Erwerbungen im Rahmen der Richtlinien der 1. Förderphase

2. Fortsetzung der thematisch-fokussierten Digitalisierung zur Unterstützung von Projekten und Forschungsvorhaben durch Bereitstellung von Digitalisaten

3. Weiterentwicklung des Fachrepositoriums, des Portals CarLi+ und Aufbau einer Metasuche für Karten und kartographische Fachliteratur

4. Einbindung alter gedruckter Bibliographien zur Kartographie in die BC mittels OCR-Verfahren

5. Ausbau der Beratungs- und Vermittlungsstelle zur Beschaffung von Geobasisdaten
Im Rahmen der Erwerbungen werden weiterhin je nach Marktsituation und aktueller Schwerpunktthemen in der Forschung neue Karten, Kartenwerke und Atlanten über alle Themen und aus aller Welt angekauft und in den Katalogen nach den bestehenden Regeln erschlossen. Dabei spielt die Erscheinungsform, ob traditionell als Druck oder digital auf Datenträgern, keine Rolle. Was seitens der Fachcommunity, anderer FIDs oder zentraler Forschungsbibliotheken benötigt wird, sich aber noch nicht im Bestand der Kartenabteilung befindet, wird über das einschlägige Händlernetz zu beschaffen versucht. Über das aktuell erworbene Kartenmaterial gibt auch die online verfügbare Neuerwerbungsliste ${ }^{9}$ Auskunft.

Für die thematisch-fokussierte Digitalisierung können auf Antrag Digitalisate von Karten aus dem Bestand der Kartenabteilung angefertigt werden. Diese werden hochauflösend gescannt und der Forschung zur Verfügung gestellt. Unterstützung erfährt dieser Service von der Beuth Hochschule für Technik Berlin, deren studentische Hilfskräfte bei Bedarf gleichzeitig erste Bearbeitungen der Kartenimages nach Vorgaben der Auftraggeber vornehmen können. Die speicherintensiven

\footnotetext{
9 sbb.berlin/5cn4p8
} 
Dateien werden über einen Server zum Herunterladen bereitgestellt. Reduzierte Versionen werden nach Erstellung von Strukturdaten in den einschlägigen Bilddatenbanken eingebunden. In diesem Zusammenhang sei auf das Projekt Kartenspeicher ${ }^{10}$ hingewiesen, das von der Verbundzentrale des Gemeinsamen Bibliotheksverbundes als Weiterentwicklung des in Jena gestarteten Kartenarchiv Plus ${ }^{11}$ betrieben wird. Ziel dieses Vorhabens ist eine schnelle graphisch aufbereitete und zeitlich einzugrenzende Recherche nach Kartenbeständen in Archiven und Bibliotheken Deutschlands. Die DGfK-Kommission Kartenkuratoren ist an der Entwicklung beteiligt, da so die von den Kuratoren verwalteten Kartenbestände unter einem gemeinsamen Dach übersichtlich recherchiert werden können.

Die bereits genannte Bibliographia Cartographica wird künftig mit dem im Aufbau befindlichen Fachrepositorium gemeinsame Wege gehen und unter den Namen CarLi+ weitergeführt werden, denn die Verwendung derselben technischen Plattform für Bibliographie und Repositorium impliziert verschiedene Vorteile. So ist einerseits die Erschließung der Dokumente im Repositorium nahezu identisch zu der seit Jahren in der Bibliographie angewandten Datenerfassung. Andererseits ist aber auch a priori eine erheblich größere Treffermenge bei der Recherche gegeben. Noch sind hierzu etliche Programmierungen vorzunehmen und die Datenmigration der alten Bibliographie-Daten in das neue System wird auch noch zu meistern sein.

Lediglich erste Konzeptionen existieren für die sogenannte Metasuche, die eine übergreifende Recherche nach Karten und Fachliteratur in mehreren Verzeichnissen wie $\mathrm{CarLi}+$, der IKAR-Altkartendatenbank, Geo-Leo, Old Maps Online oder der David-Rumsey Historical Map Collection

\footnotetext{
$\overline{10}$ http://kartenspeicher.gbv.de.

11 Christoph, Andreas u.a.: Digitalisierung - Verortung - Recherche. Neue Perspektiven für historische Karten. In: KN 66, 2016, 3, S. 115-122
}

erlauben wird. ${ }^{12}$ In weiteren Schritten ist die Erweiterung um eine Suche nach Geodaten und nach Forschungsdaten vorgesehen.

Parallel wird an der Erweiterung der Bibliographie durch die Einbindung alter gedruckter Literaturverzeichnisse zur Kartographie gearbeitet. Die Onlineausgabe der BC beinhaltet Nachweise ab 1989, als mit der elektronischen Datenerfassung begonnen wurde. Doch wurde die systematische Verzeichnung der kartographischen Fachliteratur bereits einige Jahrzehnte früher begonnen. Die von 1957 bis 1972 erschienene Bibliotheca Cartographica mit über 23.000 Titeln und ihre Nachfolgerin, die ab 1973 erschienene Bibliographia Cartographica, die bis 1988 über 40.000 Literaturhinweise angezeigt hat, werden nach dem Scannen mit entsprechenden Texterkennungsprogrammen bearbeitet und der Online-Ausgabe zugeführt. Die Library of Congress stellt darüber hinaus die Daten aus der von ihr herausgegebenen Bibliography of Cartography (5 Bände plus 2 Supplemente) zur weiteren Bearbeitung zur Verfügung und schließlich soll die Datenbank GeoPhoKa des Bundesamtes für Kartographie und Geodäsie mit ihren ca. 65.000 Titeleinträgen integriert werden. Damit dürfte dann eine umfassende Literaturdatenbank zur Kartographie entstehen.

Auch die Geodatenvermittlungsstelle kann, die in der ersten Förderphase begonnene Arbeit fortsetzen und weiter ausbauen. Die bereits auf Bundesebene aufgebaute Kooperation mit dem BKG soll insbesondere den Datentransfer für die Wissenschaft befördern. Im Fokus steht dabei u. a. die Ableitung von historischen Geodaten aus digitalisierten alten Landesaufnahmen. Aber auch die interdisziplinäre Zusammenarbeit mit anderen Fachinformationsdiensten, in denen Raumdaten zur Verarbeitung kommen, ist weiter voranzutreiben.

\footnotetext{
12 IKAR: http://ikar.staatsbibliothek-berlin. de/; GEO-LEO: https://geo-leo.de/; Old Maps Online: https://www.oldmapsonline.org/; David Rumsey Historical Map Collection: https://www.davidrumsey.com/.
}

Dabei ist die Ausgangssituation zur Beschaffung von Geodaten nach wie vor recht heterogen. Das betrifft sowohl die Herkunft, die Qualität und die Formate dieser Daten als auch die Preisgestaltung oder die Rechtslage. Die Fachcommunity soll deswegen weiterhin mit dem eingerichteten Service der zentralen Anlaufstelle für die Vermittlung und Beschaffung von Geodaten unterstützt werden. Aufgabe der Vermittlungsstelle ist die Beratung und Hilfe bei der Ermittlung des benötigten Raumausschnittes und der Festlegung verschiedener Faktoren wie Datenformat, Maßstab, Auflösung und Inhalt, die Einfluss auf die Preiskalkulation haben, um eine individuelle und passgenaue Datenlieferung auslösen zu können. Für die Verhandlungen mit den Herausgebern oder Vertriebshändlern von Geodaten wird auf die Verbindungen der Kartenabteilung zum spezialisierten Fachhandel zurückgegriffen. Die Geodaten sind abschließend durch den Auftraggeber selbst beim Herausgeber oder Händler zu erwerben. Falls keine Geodaten des benötigten Raumausschnittes erhältlich sind, kann auf den gedruckten Kartenbestand der Kartenabteilung zurückgegriffen werden, um durch eine Digitalisierung zumindest Rasterdaten anbieten zu können. Falls gewünscht, könnten in diesem Fall zur Vorbereitung weiterer Studien kartometrische Analysen, die Auskunft über die geodätische Genauigkeit der Kartengrundlage geben, angefertigt werden.

Die Serviceangebote des FID Karten sollen dabei nicht nur den Mitgliedern der engeren Fachcommunity zur Verfügung stehen, sondern allen, die sich mit der Disziplin der Geoinformation, mit Karten und Raumdaten beschäftigen, zugutekommen. Das impliziert eine engere Verzahnung mit den sonstigen Aufgaben und der Arbeitsorganisation der Kartenabteilung der Staatsbibliothek zu Berlin. So freuen wir uns auf drei weitere Jahre der Förderung zum Wohle der Kartographie.

Wolfgang Crom

Staatsbibliothek zu Berlin 


\section{Rezensionen}

Franckh-Kosmos (Hrsg.):

\section{KOSMOS UNIVERSAL ATLAS}

\section{Der Atlas für die ganze Familie}

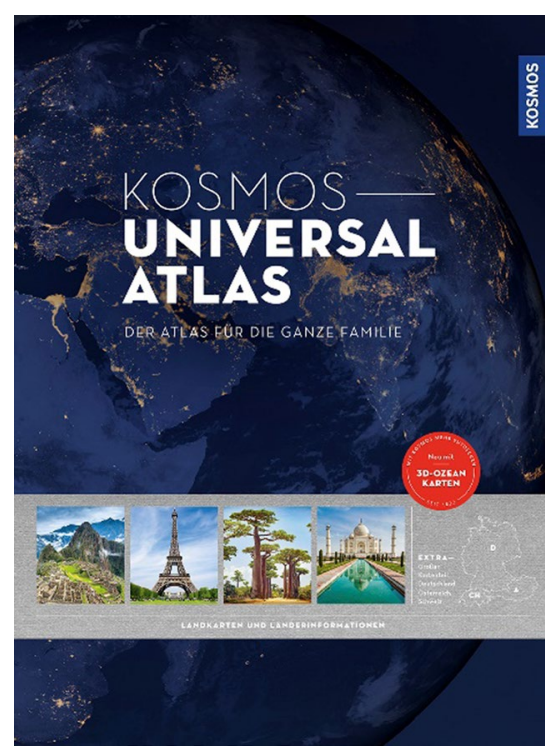

Mit 23 Farbfotos und 320 Illustrationen.

Franckh-Kosmos Verlags-GmbH \& Co. KG, Stuttgart 2019

ISBN: 978-3-440-16427-3; Einband $36 \mathrm{~cm}$ x 26,5 cm, 314 Seiten.

Auf dem Einband läuft über Vorder- und Rückseite eine „NASA Erddarstellung“": dunkelblaugraue Nachtseite der Erde mit gelblicher Beleuchtung. Auf der Vorderseite steht oben der vierzeilige Titel in weißer Versalschrift. Darunter ist durchaus werbewirksam wie ein runder, roter Aufkleber zu lesen: Neu mit 3D-Ozean Karten; darunter folgt: neben 4 Farbfotos EXTRA - Großer Kartenteil Deutschland, Österreich, Schweiz, und auf der Rückseite unten als Streifen vier kleine Kartenausschnitte der im Band benutzten Kartenarten: Weltkarten (1 : 30 Mill.), Länderkarten (1 : 4,5 Mill.), Straßen- und Reisekarten (1: 750 000), 3D-Ozeankarten ( $1: 85$ bis 1 : 20 Mill.; dazu unter EIN
WELTATLAS - IM GROSSFORMAT stehen 17 Zeilen in zwei Textblöcken und vier Anstrichen; Eigenwerbung des Verlags.

Zum vierten Mal liegt Rezensenten ein Atlasband mit überwiegend den gleichen Karten vor, einst hergestellt als Digital Chart of the World der ESRI Inc. In Deutschland wohl zuerst benutzt in Der große wissen.de Weltatlas" (2002) im Wissen Media Verlag; damals abgedruckt im Maßstab 1 : 3 Mill.; dann weiterbenutzt im Der Brockhaus Weltatlas, der in 9. Aufl. 2012 erschien (s. Rez. in KN 2013/4, S. 228-230), zuletzt in Der große KOSMOS Weltatlas 2014 mit Länderkarten 1 : 4 Mill. und nun unter dem Schlagwort Universal Atlas, was er nach dem Verständnis bisheriger Universal-Atlanten und deren gängigen Definitionen bestimmt nicht ist (vgl. bei S. Schulz, 2006, S. 12). Tatsächlich ist die Struktur einfach, fast elementar. Der Band besteht nach der Gliederung des Inhaltsverzeichnisses aus den Abschnitten Die Erde (S. 2-22), basierend auf einer Erdkarte 1:80 Mill., ausgeführt physisch und politisch im Van-der-Grinten-Entwurf, einschließlich acht auf $1: 30$ Mill. bis $1: 10$ Mill. daraus vergrößerten Erdteilblättern, gefolgt vom Hauptteil, den in 6 Erdteilblocks gegliederten Länderkarten 1 : 4,5 Mill. (S. 24-164), dem neu hinzugefügten Block Ozeane mit einer Karte Weltmeere (sic!) und 7 Blättern der Ozeane sowie polständig Südpolarmeer (S. 178/79) und Nordpolarmeer $1: 20$ Mill. (S. 180). Der letzte Kartenblock (S. 182-204) beinhaltet D-A-CH physisch und politisch in $1: 4$ Mill. und 12 Karten 1:750 000. Es folgen Länderinformationen als zehnspaltige Tabelle mit einer Kopfleiste aus Farbfotos (S. 205-214), das Namenverzeichnis (S. 215-297) und separat Namenverzeichnis Deutschland, Österreich, Schweiz
(S. 297-313); auf S. 314 folgen noch Bildnachweis und Impressum.

Der vordere Vorsatz zeigt auf geschummerten Landflächen ohne Kartennetz in Rot die Blatteinteilung 1 : 30 Mill. / 1 : 4,5 Mill. und eingefügt unter Afrika für D-A-CH 1 : 750 000; auf dem hinteren Vorsatzblatt sind 196 Flaggen der Erde angeordnet, was natürlich Flaggen der souveränen Staaten heißen müsste; hier erstmalig seit meiner ersten Begegnung mit einer solchen Flaggentafel im Lehrbetrieb 1942 nicht in Rechteckform, sondern gewellt im Sandsturm: nämlich schattiert, was zu verschmutzten, manchmal kaum deutbaren Farben der Fahnentüchern geführt hat. Die Anzahl entspricht der Flaggentafel im Fischer Weltalmanach 2019, aber in teilweise geänderter Reihung aufgrund teilweise anderer Ländernamen (z. B. statt China, Rep. hier Taiwan, statt Myanmar hier Birma).

Netzentwurf und Kartenbild der Erd-, Erdteil- und Länderkarten sind wiederholt kritisch beleuchtet worden. Die Anwendung des flächentreuen Netzentwurfs nach Albers für die Länderkarten 1 : 4,5 Mill. und des winkeltreuen Entwurfs von Lambert bei den Straßen- und Reisekarten $1: 750000$ ist auch im vorliegenden Atlas durchaus zweckentsprechend. Neu ist die weitere Verkleinerung der Kartenfläche um 20\%. Gleichzeitig wurde der Kartenspiegel der Seiten bzw. Blätter von $29,5 \mathrm{~cm}$ x 23,5 cm auf $32,5 \mathrm{~cm}$ x 24,5 cm vergrößert. Daraus folgt großenteils ein neuer Blattschnitt bzw. eine weitere Ausweitung der Überlappungen und auch Erweiterung angrenzender Meeresgebiete, was insgesamt keine Verbesserung bedeutet. Die Gesamtkartenfläche $1:$ 4,5 Mill. beträgt jetzt $10,4 \mathrm{~m}^{2}$, was 208 Mill. $\mathrm{km}^{2}$ entspricht; darunter 100 Mill. $\mathrm{km}^{2}$ Landflächen. Der Rest entfällt auf angrenzende Meeresgebiete und die 
Blattüberlappungen. Dafür macht die Maßstabsverkleinerung das Kartenbild insgesamt noch schwerer lesbar, vieles ist ohne Lupe für Normalsichtige nicht mehr erkennbar, insbesondere die kleinsten Ortsringe. Nachteilig ist auch der Wegfall der Gradnetzunterteilung im Rand. Bei den als Ausschnitte aus der Erdkarte gebildeten Erdteilkarten ist damit die letzte Erkennbarkeit des gleitenden Maßstabes weggefallen; eine Verortung von Objekten in den großen, schiefschnittigen Netzmaschen ist dadurch nicht mehr möglich. Blickfang bleibt die Europakarte (S. 6/7) mit der Abbildung Grönlands (2 Mill. $\mathrm{km}^{2}$ ) in der Größe von ganz Europa (10 Mill. $\mathrm{km}^{2}$ ) und einem darüberstehenden Längenmaßstab für $1: 30$ Mill. bei real ca. 1: 20 Mill. im Süden und 1:10 Mill. im Norden. Als Breite Grönlands in $80^{\circ} \mathrm{N}$ weist der Längenmaßstab statt $920 \mathrm{~km}$ real auf S. 22 hier stolze 3800 km aus!

$\mathrm{Zu}$ den 3D-Ozeankarten heißt es: Die eindrucksvolle dreidimensionale Darstellung der sieben Weltmeere, zeigt die faszinierenden Landschaften und Gebirge unter Wasser. Den Begriff Landschaft für benannte Meeresbodenformationen zu benutzen ist wenig sinnvoll. Eine graue Schattierung des untermeerischen Reliefs in einer angenommen „Beleuchtung“ von links oben ohne Beibehaltung der Tiefenlinien und ohne Gradnetz kann keine realen Vorstellungen erzeugen. Polarkarten ohne Richtung gebendes Netz sind ohnehin ein Unding. Die eingeschriebenen Benennungen meist in deutscher Sprache sind überdies in den dunklen Schattentönen kaum lesbar; damit ist das Ganze ein deutungsloses Fleckenmuster. Dem Block der Karten 1 : 750000 sind eine Physische und eine politische Übersicht 1 : 4 Mill. vorangestellt. Auf der linksstehenden Physischen Karte endet die Höhenschichtenfärbung an den Staatsgrenzen; in den umliegenden Regionen setzen sich bis zum Kartenrand nur die Strichelemente einschließlich der Ortssignaturen und die Reliefschummerung fort. Eine merkwürdige Lösung! Immerhin macht dieses Blatt den Lesbarkeitsverlust des großen Blocks der Länderkarten in jetzt $1: 4,5$ Mill. (Mitteleuropa S. 27) deutlich. Die rechts gegenüberstehende Politische Übersicht vermittelt mit violettem Grenzband und hell bis dunkel abgestuften Grüntönen die Ländergliederung von Deutschland und Österreich, nicht aber die Kantone der Schweiz, die nur im großen Maßstab mit auffällig breitem Grenzband und Kantonsnamen überdeutlich hervortreten (S. 200/201). In dem eigenwilligen Kartenbild der drei mitteleuropäischen Staaten, zu dem es keine eigene Zeichenerklärung gibt, fallen insbesondere die roten Flächen um hohle quadratische Siedlungssignaturen auf. Man kann sie als „bebaute Fläche“ deuten. Aber ca. $50 \mathrm{~mm}^{2}$ bei Osterode bedeuten dann rund $25 \mathrm{~km}^{2}$ für 27 Tsd. Ew.; etwa mit gleicher Größe ist Erfurt wiedergegeben; dafür Gera deutlich kleiner. Noch verheerender wirkt sich allerdings aus, dass alle Orte ohne Rotflächen als graue hohle Löcher kaum erkennbar sind. Dasselbe gilt für Eisenbahnen, während die roten Straßen aufdringlich hervortreten.

Die 8 Seiten Länderinformation sind als zehnspaltige Tabelle, geordnet nach Erdteilen, mit einer Kopfleiste aus Fotos ausgeführt. Für jeden Staat werden genannt: Land, unbenannt Flagge, Ländername, worunter die amtliche Staatsbezeichnung in Deutsch zu verstehen ist, Autokennzeichen, womit das Staatenkürzel der Kfz-Kennzeichen gemeint ist; es folgen Hauptstadt/Regierungssitz, Fläche (in $\mathrm{km}^{2}$ ), Anzahl der Einwohner, ohne Jahresangabe (meist für 2010), Einwohnerzahl pro $\mathrm{km}^{2}$ Landfläche, Landessprache, Währung und Zeitzone. Unüblich schließt die Fläche Frankreichs mit $668763 \mathrm{~km}^{2}$ die zur EU gehörenden Übersee-Departements ein; bisher galt immer ohne diese $543965 \mathrm{~km}^{2}$.

In den beiden Namenverzeichnissen mit zusammen 87 Tsd. Einträgen werden für die Objektarten 18 Piktogramme und für Nationen und Gebiete die internationalen ISO Alpha-3 Codes benutzt, gefolgt von Seiten- und Suchfeldangabe. Nach Stichproben werden bei überlappender Darstellung die Namen mit beiden Suchfeldern getrennt aufgeführt.

Nach Jahrzehnten der Nutzung liegt nunmehr eine mit minimiertem Aufwand erzeugte Ausgabe vor: Auf nochmals verkleinerter Kartenfläche ein nur noch schwer lesbares Kartenbild, ohne jede Erläuterung (Benutzerhinweise beschränkt sich auf Begriffserklärungen zu Kartenzeichen auf stummen Kartenbildern von Kalifornien), dazu Minimierung der Blattrandangaben mit Einsparung der zur Verortung des Karteninhaltes notwendigen Gradnetzunterteilung. Zugleich Fortschreibung der alten Fehler, wie falsche Maßstabsleisten auf den Erdteilkarten. Besonders gravierend bleibt mit Blick auf den angestrebten Zweck die völlige Vernachlässigung der Menschheit auf diesem Planeten mit dem hervorstechenden Merkmal ihres rasanten Wachstums. Die Auswahl dargestellter Städte und die mit Wechsel zwischen Verdoppelung und Verfünffachung der Gruppenbreite schlecht gewählten Größenklassen können nur ein unzulängliches Bild der Bevölkerungsverteilung vermitteln. Ebenso ist in den Länderinformationen nur eine zeitlich zurückliegende Einwohnerzahl für Staaten absolut statisch. Für zwei etwa 30 Jahre auseinanderliegende Werte wäre ohne Mehraufwand Platz gewesen. Zu den wichtigsten Basisdaten gehört zweifellos die Verdeutlichung des Bevölkerungswachstums. Seit ca. 3 Generationen wächst die Erdbevölkerung stündlich um eine Kleinstadt von 10 Tsd. Einwohnern, täglich damit um eine neu zu bauende und zu versorgende Großstadt. Von Woche zu Woche nimmt die Bevölkerung um mehr als 1 Mill. Menschen, jährlich um etwa 80 Mill. zu, was damit etwa der Bevölkerung Deutschlands entspricht. 
Der in randscharfem, passgenauem Druck ausgeführte Band mit stabilem Einband wird zu einem erstaunlich niedrigen Preis vertrieben. Da ist es vorstellbar, dass für redaktionelle und kartographische Arbeiten nur sehr begrenzte Mittel zur Verfügung standen. Aus dieser Sicht verständlich, dass bei den Kartenblocks nach Inhalt und Ausführung die genannten Mängel zustande kamen. In dieser Form kann der Band nur einfache Nachschlagefunktionen, sicher auch für die ganze Familie, erfüllen. Ein für jede Gelegenheit perfektes Nachschlagewerk, wie es in der rückseitigen Anpreisung des Werkes auch heißt, ist es in der vorgelegten Form nicht.

Werner Stams, Radebeul

\section{Neuerscheinungen}

Bolashvili, N.; Dittmann, A.; King, L.; Neidze, V., (Hrsg.)

\section{National Atlas of Georgia}

Franz Steiner Verlag; Stuttgart 2018, erste Aufl., geb., 138 Seiten, englisch, EUR 196,00 [D]

ISBN-10: 3515120572

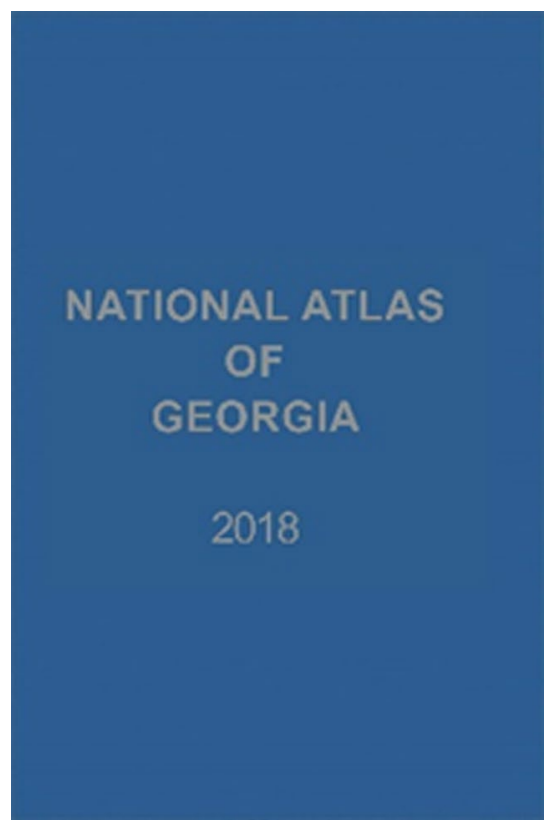

Hinz, S., Vögte, T., Wursthorn, S. con terra GmbH (Hrsg.): (Hrsg.):

\section{Digitale Bildverarbeitung und Geoinformation.}

Datenfusion unter geometrischen, physikalischen und semantischen Aspekten

Wichmann, VDE Verlag, Berlin, 5. vollst. neu bearbeitete Auflage, 2019, 341 Seiten, 170 × $240 \mathrm{~mm}$, Broschur, EUR 56,00 [D], ISBN 978-3-87907-638-3, E-Book: ISBN 978-3-87907-639-0

Stefan Hinz/Thomas Vögtle/Sven Wursthorn (Hrsg.)

\section{Digitale Bildverarbeitung und Geoinformation}

Datenfusion unter geometrischen, physikalischen und semantischen Aspekten

5., vollstảndig neu bearbeitete Auflage

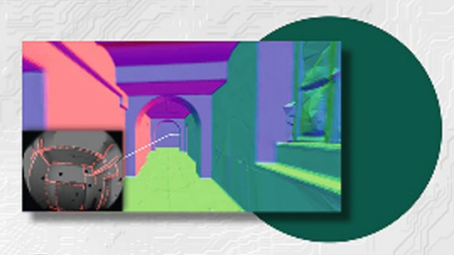

Wichmann

\section{FME Desktop}

\section{Das deutschsprachige Handbuch für Einsteiger und Anwender}

Wichmann, VDE Verlag, Berlin, 2., neu bearbeitete und erweiterte Auflage 2018, 442 Seiten, 170 x 240 $\mathrm{mm}$, Broschur, EUR 64,00 [D], ISBN 978-3-87907-648-2, E-Book: ISBN 978-3-87907-649-9

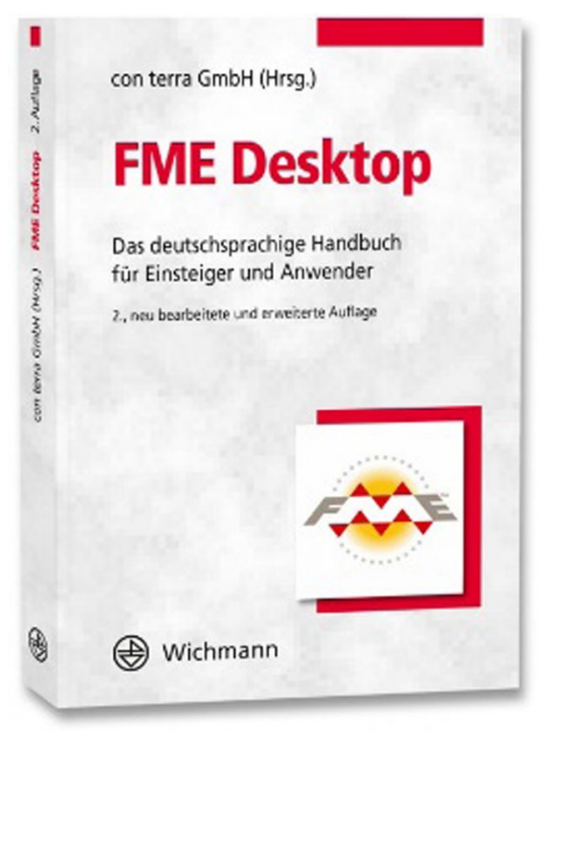


Bischoff, M.; Lüpkes, V.; Crom, W. Ette, O., Lubrich, O. (Hrsg.):

(Hrsg.):

\section{Kartographie der Frühen Neuzeit}

\section{Weltbilder und Wirkungen}

Jonas Verlag F., Ilmtal 2015, Band 5 Studien zur Kultur der Renaissance, 259 S., Hardcover, Format (B x L): 17.9 x $24.6 \mathrm{~cm}, 201 \mathrm{Abb} .$, EUR 30,00 [D], ISBN 978-3-89445-516-3

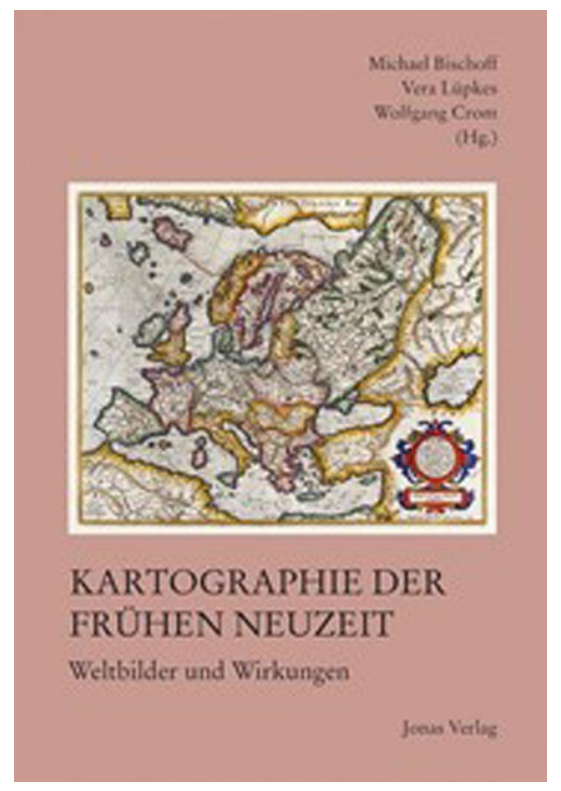

\section{Alexander von Humboldt}

\section{Kosmos - Entwurf einer physischen Weltbeschreibung}

Die Andere Bibliothek, Berlin, 2014, 941 S., Foliobände, EUR 128,00 [D], ISBN: 9783847700142

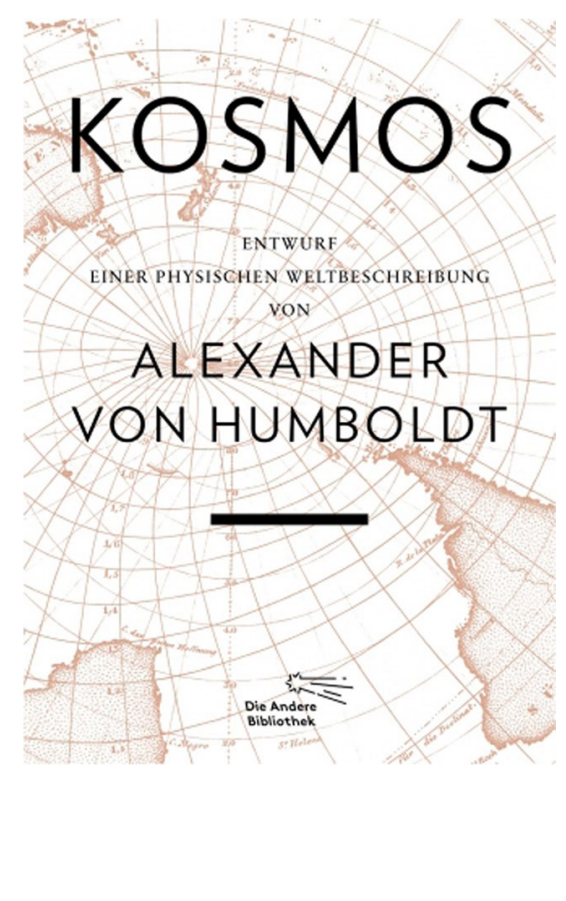

\section{Geoinformation aktuell / Business News}

\section{Projektstart: „BeetScan“ unterstützt den Zuckerrübenanbau durch Auswertung von Satellitendaten}

\section{Julius Kühn-Institut (JKI) am Forschungskonsortium beteiligt}

Bei dem neuen Projekt „BeetScan“ geht es nicht etwa um Blumen-Beete, sondern um „sugarbeets“, englisch für Zuckerrüben. Ziel ist, die derzeit verfügbaren Satellitendaten sinnvoll auszuwerten, um so die Zuckerrübenanbauer in Deutschland zu unterstützen.
Das Bundesministerium für Wirtschaft und Energie (BMWI) fördert das Projekt über zwei Jahre mit 330.000 Euro. Die technische und methodische Umsetzung berieten die Partner des „BeetScan“-Konsortiums auf ihrem Kick-off-Meeting im April 2019 beim Raumfahrtmanagement des Deutschen Zentrums für Luft- und Raumfahrt in Bonn. Zum Konsortium gehören die Nordzucker AG (Koordinator), die Firma m2Xpert, die Arbeitsgemeinschaft zur Förderung des Zuckerrübenanbaus in Norddeutschland e. V. sowie das Julius Kühn-Institut (JKI).
Die Europäische Union stellt mit dem Copernicus Programm Satellitendaten in bisher nicht gekannter Qualität und zeitlicher Verfügbarkeit kostenfrei zur Verfügung. Jeder Ort in Deutschland wird alle 1-3 Tage von Radarsatelliten und alle 3-5 Tage von optischen Satellitensystemen überflogen. Damit sei ein sehr gutes Monitoring landwirtschaftlicher Flächen möglich, da Veränderungen in den Pflanzenbeständen schnell erkannt werden könnten, erklärt Dr. Holger Lilienthal vom Julius Kühn-Institut in Braunschweig. So wäre es für die Anbauer von Zuckerrüben möglich, sich einen Überblick 


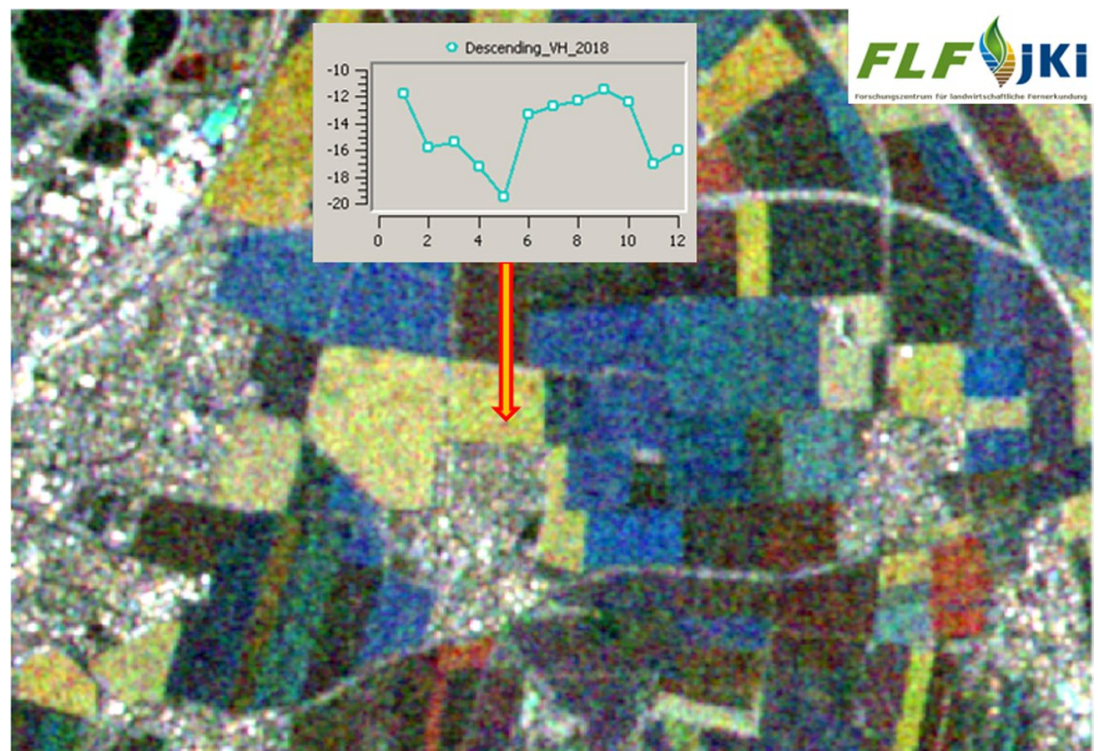

Zuckerrübenschlag (gelb) im Radarbildkomposit von Sept/Okt/Nov 2018. Für jeden Bildpunkt kann eine Signatur gemessen werden, die die Veränderung des Radarsignals im Jahresverlauf anzeigt, um charakteristische Termine (Aussaat oder Ernte) zu bestimmen. (C FLF am JKI

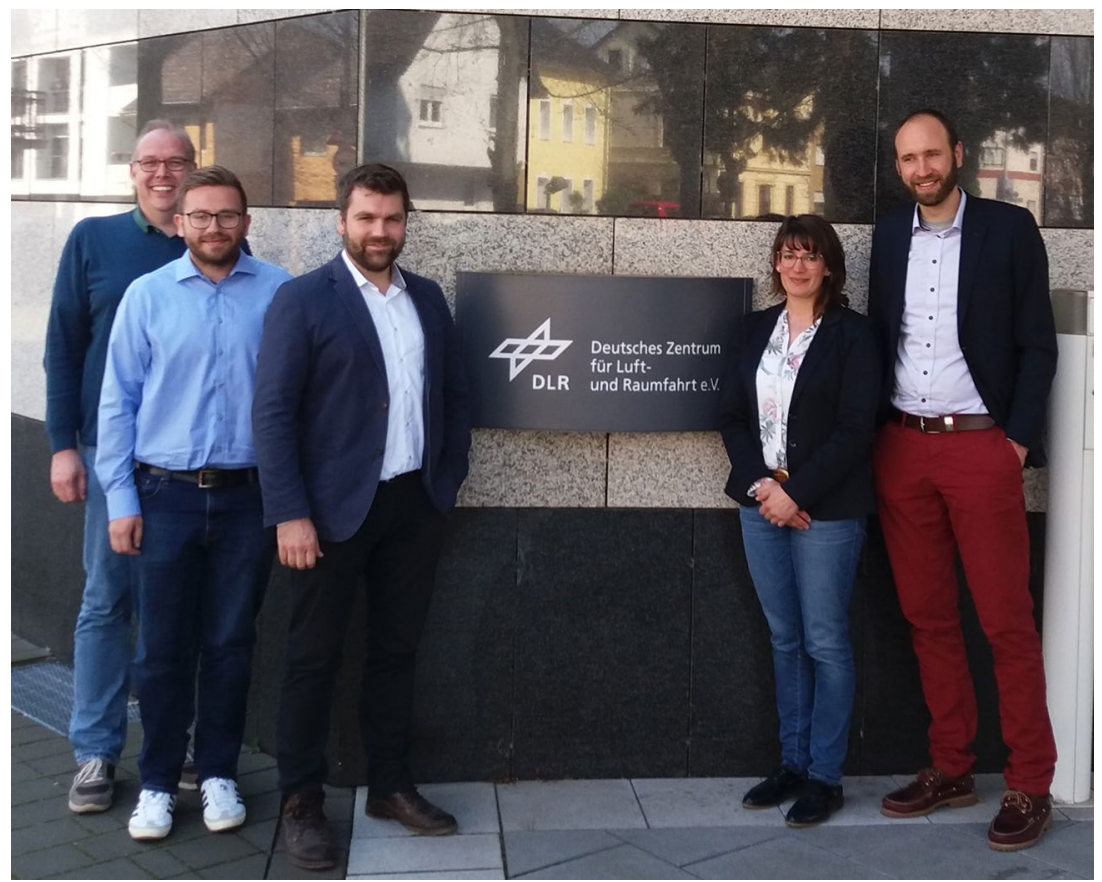

Gruppenfoto des Kick-off-Meetings. (c) FLF am JKI

über den aktuellen Zustand ihrer Felder zu verschaffen. Zusätzlich könnten auch wichtige Zeitpunkte wie beispielsweise Aussaat, Auflaufen der ersten Pflanzen, Bestandsschluss oder der Erntetermin schlaggenau erfasst werden. Aus diesen Daten wiederum ließen sich zu
Datenmengen eröffneten sich auch neue Auswertungsverfahren aus dem Bereich der künstlichen Intelligenz, wie zum Beispiel maschinelles Lernen, die im Projekt BeetScan getestet werden sollen.

- Informationen: Forschungszentrum für landwirtschaftliche Fernerkundung (FLF) am JKI, c/o JKI-Fachinstitut für Pflanzenbau und Bodenkunde, Bundesallee 69, D-38116 Braunschweig; Internet: flf.julius-kuehn.de

\section{BKG-Service für die Bundesverwaltung}

\section{Fernerkundungsdaten für jedes Gebiet auf der Erde}

Das Bundesamt für Kartographie und Geodäsie (BKG) hat ein Verfahren entwickelt, mithilfe dessen sich Fernerkundungsdaten bedarfsbezogen bereitstellen lassen. Dank des sogenannten Mosaik-Verfahrens steht ab sofort ein komplettes, nahezu wolkenfreies und qualitativ hochwertiges Deutschlandmosaik aus Sentinel-2-Daten aus 2018 als Open Data zur freien Nutzung für jedermann zur Verfügung.

Derzeit werden für die fernerkundliche Produktion bevorzugt optische Satellitenbilddaten in der Bundesverwaltung eingesetzt. Hierfür ist das Mosaik-Verfahren eine wichtige Methode. Es wurde mit EU-finanzierten Sentinel-2-Daten der europäischen Erdbeobachtungsinitiative Copernicus aufgebaut und kann für jeden optischen Datensatz (inkl. Luftbilder) angewendet werden. Das BKG stellt mit dieser Methode für die Bundesverwaltung einen Service zur Verfügung, über den sich optische Fernerkundungsdaten für jedes Gebiet auf der Erde fachlich und bedarfsorientiert aufbereiten lassen.

Die Produkte kommen u. a. in der Flugsicherung oder bei der Analyse der Landbedeckung und Landnutzung in ihrer zeitlichen Veränderung zum Einsatz. Dadurch leistet das BKG einen 
unterstützenden Beitrag zur zivilen Sicherheit und für verschiedene nationale Berichtspflichten der Bundesverwaltung (z. B. Umweltberichte, Statistikberichte, Berichte zur Veränderung der Lebensverhältnisse).

Das kostenfreie Deutschlandmosaik aus Sentinel-2-Daten aus dem Jahr 2018 hat eine Bodenauflösung von zehn Metern. Es wird als sogenannter Web-Map-Service (WMS) bereitgestellt. Der Webdienst bietet drei verschiedene Farbkomposite (ein RGB-Echtfarbenbild und zwei Falschfarbendarstellungen) für verschiedene Anwendungszwecke. Eine jährliche Aktualisierung ist vorgesehen.

- Informationen: Bundesamt für Kartographie und Geodäsie (BKG), RichardStrauss-Allee 11, D-60598 Frankfurt am Main; Internet: www.bkg.bund. de; Weitere Informationen für Bundesnutzer im Internet: www.bkg.bund. de/DLZ; Weitere Informationen zum Open-Data-Dienst Sentinel-2-DE im Internet: www.geodatenzentrum.de/ geodaten/gdz?l=down_sentinel2

\section{BKG-Jahresbericht 2018 ist ab sofort verfügbar}

Das Bundesamt für Kartographie und Geodäsie (BKG) hat seinen Jahresbericht 2018 online veröffentlicht. Der Bericht steht auf den Webseiten des BKG zum Download bereit und wird ebenfalls in Druckform erhältlich sein. Auch im vergangenen Jahr 2018 hat das BKG wieder zahlreiche Projekte angestoßen, begleitet oder erfolgreich abgeschlossen, worüber das BKG gerne informieren möchte. Neben einem Jahresrückblick in Kurzform und den ausführlichen Fachthemen wurde die Rubrik „Zahlen - Daten - Fakten 2018“ zusammengestellt. Diese beinhaltet eine Auswahl interessanter und wissenswerter Angaben auf einen Blick aus der täglichen Arbeit im BKG.
- Informationen: Bundesamt für Kartographie und Geodäsie (BKG), RichardStrauss-Allee 11, D-60598 Frankfurt am Main; Internet: www.bkg.bund.de; Jahresbericht im Internet: www.bkg. bund.de/jahresberichte

\section{digital@bw macht Kartendaten mobil nutzbar}

\section{„Geoportal plus" online}

Anlässlich der Aktivierung des „Geoportals plus“ im März 2019 sagte der Minister für Ländlichen Raum und Verbraucherschutz, Peter Hauk MdL, in Stuttgart, dass ab sofort über das Geoportal Baden-Württemberg der einfache Zugriff auf hochwertige Kartendaten des Landes und der Kommunen möglich sei. Gleichzeitig könnten die Daten über smarte Portalfunktionen integriert werden. Mit der plus-Version würde dem Wunsch der Bürgerinnen und Bürger nachgekommen werden, amtliche Geodaten aus allen Bereichen der Verwaltung auch über mobiles Internet mit wenigen Klicks zu erhalten. Zugleich hätten Behörden und Unternehmen in Baden-Württemberg nun die Möglichkeit, die im Geoportal verfügbaren Daten zusammen mit eigenen Daten in eigenen Webauftritten öffentlichkeitswirksam zu nutzen.

Das Geoportal sei vom Landesamt für Geoinformation und Landentwicklung im Zuge der Digitalisierungsoffensive der Landesregierung digital@ bw als innovativer Digitalbaustein für Kartendaten weiterentwickelt worden. Die neuen Funktionen würden kostenlos bereitgestellt, so der Minister.

Das Geoportal sei jetzt auch mit dem Smartphone und dem Tablet aufrufbar. Mittels der in den mobilen Geräten integrierten GPS-Sensoren könne das Kartenbild des Geoportals automatisch auf den Standort zentriert werden. So stünden aktuelle Kartenund Luftbilddaten für die Bürgerinnen und Bürger zu jeder Zeit an jedem Ort bereit, erklärte der Minister.

Mit einem Web-Generator können Behörden und Unternehmen die im Geoportal verfügbaren Karten unmittelbar in eigene Webauftritte auf Knopfdruck einbinden. Kombiniert mit eigenen Daten ergeben sich für Banken, Immobilienfirmen und andere Unternehmen innovative Möglichkeiten, die amtlichen Daten für neue Geschäftsmodelle und für interne Anwendungen zu nutzen. Kommunen können beispielsweise Bebauungspläne zusammen mit Wasser- oder Naturschutzgebieten unmittelbar in ihrem Webauftritt darstellen und so die Bürgerbeteiligung unterstützen.

Mit dem Geoportal plus des Landesamts stünde erstmals eine einfach integrierbare Kartenkomponente samt hochwertiger Geodaten kostenlos zur Verfügung, die Qualität und Flexibilität mit Werbefreiheit und Datensicherheit verbinde, betonte Minister Hauk.

Das Geoportal Baden-Württemberg macht Karten und raumbezogene Daten aus allen Bereichen der Verwaltung für die Öffentlichkeit zentral im Internet nutzbar. Das Landesamt für Geoinformation und Landentwicklung (LGL) hat das Geoportal im Auftrag des Ministeriums für Ländlichen Raum und Verbraucherschutz (MLR) entwickelt und ausgebaut.Das Geoportal bildet den Zugangsknoten zur Geodateninfrastruktur Baden-Württemberg (GDI-BW). Die GDI-BW wird gemeinsam von Land, Kommunen, Wirtschaftsverbänden und Hochschulen getragen.

- Informationen: Fraunhofer-Institut für Graphische Datenverarbeitung IGD, Fraunhoferstraße 5, D-64283 Darmstadt; Internet: www.igd.fraunhofer. de; Geoportal Baden-Württemberg im Internet: www.geoportal-bw.de 


\section{Digitale Weltkarte}

Die Karlsruher DDS Digital Data Services GmbH hat Ende März 2019 einen Kooperationsvertrag mit dem niederländischen Unternehmen AND geschlossen und bietet nun die ,Digitale Geographie AND“ für alle Länder der Welt zu günstigen und individuell zugeschnittenen Konditionen an. Dabei werden auch Länder wie China abgedeckt.

China als bevölkerungsreichstes Land der Welt hat eine dynamische Wirtschaftsentwicklung hinter sich, die zu wachsenden Ex- und Importen geführt hat. Deutschland ist dabei bis heute einer der führenden europäischen Handelspartner der Volksrepublik: Knapp ein Drittel des gesamten Handelsvolumens der EU mit China entfällt auf Deutschland.

Dabei unterliegen deutsche Unternehmen in China nach wie vor zahlreichen Einschränkungen und Schwierigkeiten. Laut Andreas Lehr, Mitarbeiter der DDS Digital Data Services $\mathrm{GmbH}$, zähle zu Letzteren auch, dass die Verfügbarkeit digitaler Informationen, die für ein erfolgreiches Business unerlässlich seien, teilweise ungenügend sei. Denn wer in China aktiv sein wolle, brauche Geodaten, so Lehr.

Der Karlsruher Datendienstleister DDS hat nun die Produkte des niederländischen Unternehmens AND zu guten Konditionen im Angebot. Dazu gehört vor allem die „Digitale Geographie AND“, eine digitale Weltkarte, die in verschiedenen Maßstäben alle Gebiete der Welt abdeckt. Das gelte nicht nur für China, sondern auch für zahlreiche andere Schwellen- und Entwicklungsländer, in denen eine Beschaffung von homogenen und lizenzrechtlich bedenkenlos verwendbaren digitalen Straßennetzen oft schwierig sei, erklärt Andreas Lehr.

Die Daten eignen sich vor allem für strategische Routinganwendungen und Kartendarstellungen. Für die wichtigsten internationalen Märkte sind sie im Maßstab 1:250.000 lieferbar, für viele andere Länder der Welt mindestens im Maßstab 1:2.000.000. Enthalten sind unter anderem Ländergrenzen, Ortspunkte, Eisenbahnlinien, Points of Interest (POIs) und Bebauungsflächen. Die Angaben zum Straßennetz umfassen außerdem die Straßennamen mit zusätzlichen Attributen wie Straßenkategorie, Richtung und Einbahnstraßen.
DDS berät beim Einsatz der ANDDaten und kann dank des Kooperationsvertrags ihren Kunden wunschgemäße Datenpakete schnüren und individuelle Lizenzvereinbarungen anbieten.

- Informationen: DDS Digital Data Services GmbH, Stumpfstraße 1, D-76131 Karlsruhe; Internet: www. ddsgeo.de

\section{Globale TanDEM-X-Waldkarte verfügbar}

Wälder sind die Lunge der Erde: Sie helfen die Treibhausgaskonzentration in der Atmosphäre zu reduzieren und damit der globalen Erwärmung entgegenzuwirken, bieten Schutz und Ressourcen für Menschen, Tiere und Pflanzen - und sie gehen in alarmierendem Ausmaß verloren. Wie der Blick aus dem All verrät, ist die Landmasse der Erde heute zu rund einem Drittel von Wäldern bedeckt. Dabei fehlt bereits mehr als die Hälfte des weltweiten Bestands, die der Abholzung insbesondere seit Mitte des 20. Jahrhunderts zum Opfer gefallen ist. Um den aktuellen Zustand sowie die Entwicklungen des "grünen Organs" genau

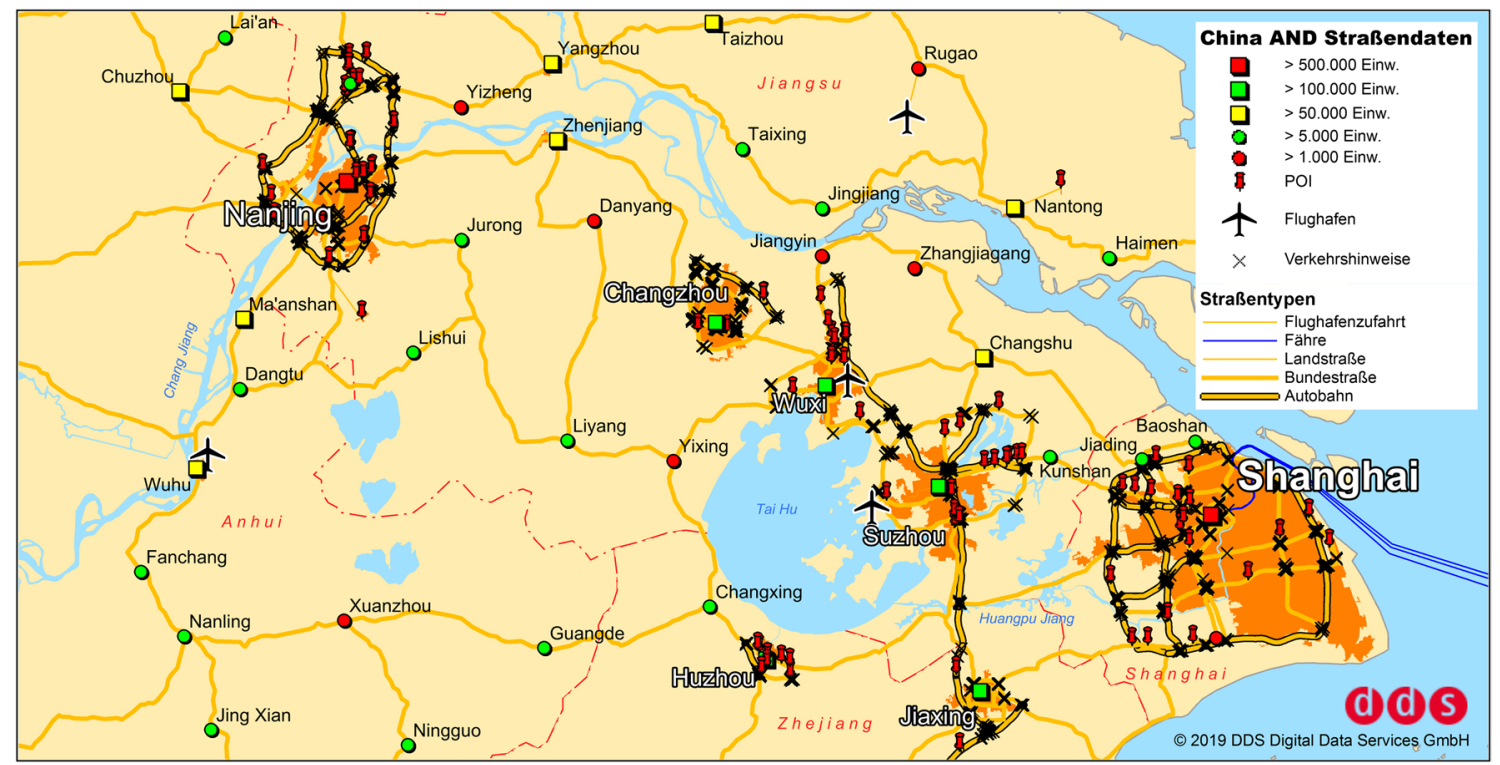


beobachten, bewerten und schützen zu können, hat das Deutsche Zentrum für Luft- und Raumfahrt (DLR) einen besonderen Datensatz erstellt: die globale TanDEM-X-Waldkarte. Dazu wurden interferometrische Daten genutzt, die für das globale Höhenmodell der deutschen Radarsatellitenmission TanDEM-X aufgenommen wurden, und Algorithmen aus dem Bereich der künstlichen Intelligenz zur globalen Datenverarbeitung entwickelt. Diese wurden für verschiedene Waldtypen anhand von Baumhöhen, Dichte und Struktur optimiert. Das Ergebnis ist eine Karte, die bei einer Auflösung von 50 Metern die Ausdehnung bewaldeter Flächen darstellt. Die globale TanDEM-X-Waldkarte des DLR steht wissenschaftlichen Nutzern ab sofort frei zur Verfügung.

Radarsatelliten können unabhängig von Wetter oder Tageszeit Aufnahmen erstellen - ein besonderer Vorteil bei der Kartierung von tropischen Wäldern, die meist von Wolken bedeckt sind. Die TanDEM-X-Waldkarte schließt bisherige Datenlücken und liefert erstmals einen einheitlichen Überblick der Regenwälder in Südamerika, Südostasien und Afrika. Die Erkenntnisse sind für Behörden und Wissenschaftler gleichermaßen bedeutsam, da diese Gebiete vor illegaler Abholzung geschützt und als mächtige Kohlenstoff-Speicher erhalten werden müssen.

Anhand der neuen Karte lässt sich entsprechend auch die Biomasse-Konzentration von Wäldern genauer bestimmen - ein Schlüsselfaktor im globalen Kohlenstoffkreislauf. Die globale TanDEM-X-Waldkarte schafft damit eine wichtige Datengrundlage für Forschungen zum globalen Wandel und bietet darüber hinaus auch vielfältige Anwendungsmöglichkeiten in der Landwirtschaft, Forstwirtschaft, Regionalentwicklung sowie der Raumplanung. In Hinblick auf die gesellschaftlichen Herausforderungen des globalen Wandels lassen sich schließlich auch genauere Vorhersagen und geeignete Maßnahmen ableiten.

Das DLR-Institut für Hochfrequenztechnik und Radarsysteme hat für das Projekt insgesamt mehr als 400.000 Datensätze verarbeitet, die im Zeitraum von 2011 bis $2015 \mathrm{im}$ Rahmen der TanDEM-X-Mission aufgenommen wurden. Um aus den riesigen Datenmengen die gewünschte Information "Wald" zu gewinnen und klassifizieren zu können, haben die Radarexperten spezielle Algorithmen entwickelt, die zuerst jede einzelne Aufnahme individuell auswerten und anschließend zu einer globalen Karte zusammenfügen. Diese Algorithmen basieren auf maschinellem Lernen aus dem Bereich der künstlichen Intelligenz und können in der Zeitschrift "Remote Sensing of Environment" (Volume 205, Februar 2018) nachgelesen werden. Künftig können so auch neue Satellitendaten ausgewertet und beispielweise in Form von Zeitreihen-Analysen mit der TanDEM-X-Waldkarte verglichen werden.

Um die gerechneten Ergebnisse zu validieren und die Waldflächen mit noch höherer Genauigkeit von den Nicht-Waldflächen abgrenzen zu können, nutzen die Entwickler zusätzliche Fernerkundungsdaten. Dazu gehören insbesondere der "Global Urban Footprint", eine globale Karte von Siedlungsgebieten die am Earth Observation Center (EOC) des DLR erstellt wurde, sowie die Gewässerkartierung der Climate Change Initiative der ESA. Die globale TanDEM-X-Waldkarte wird im Deutschen Satellitendatenarchiv des EOC verwaltet und den Nutzern bereitgestellt. Das Deutsche

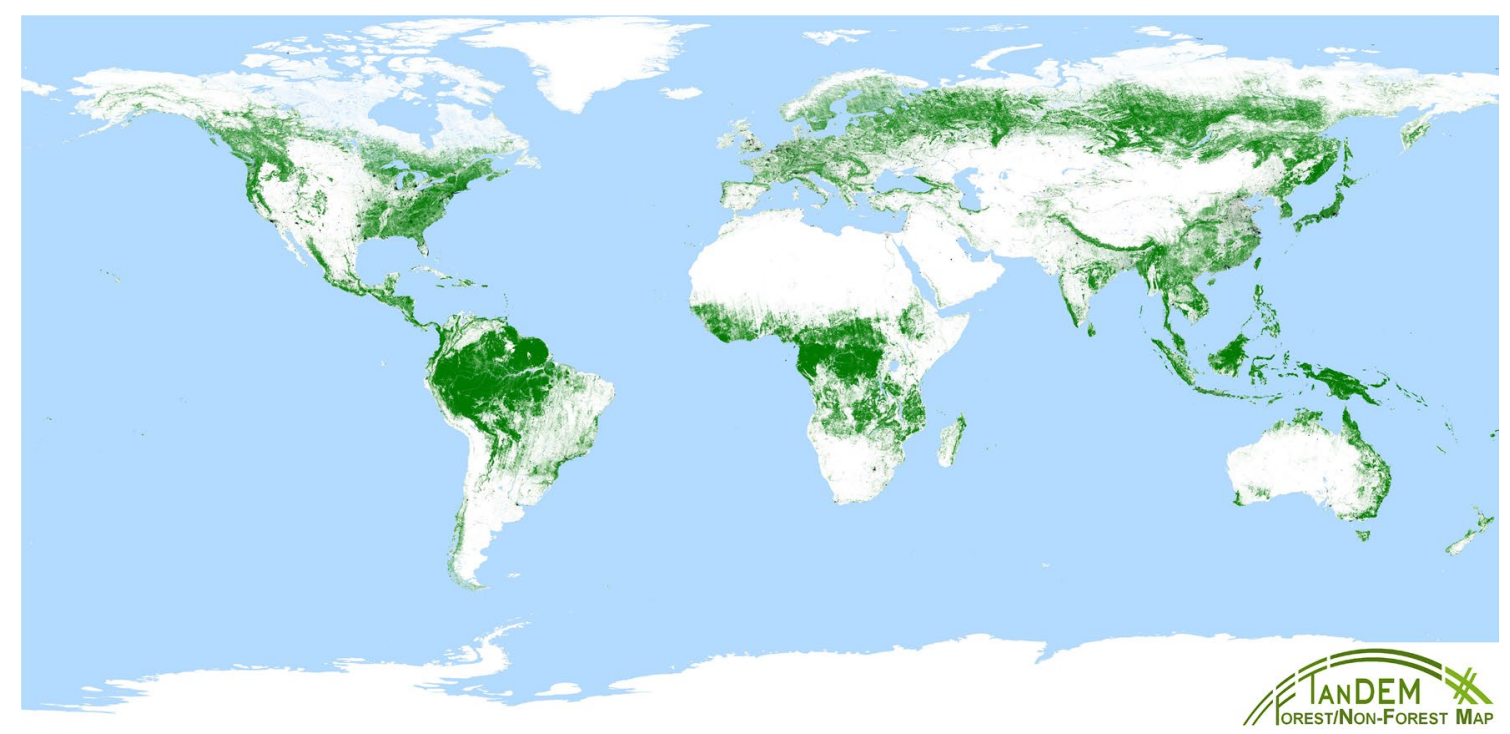

Die globale TanDEM-X Waldkarte des DLR wurde aus interferometrischen Radardaten abgeleitet und steht für wissenschaftliche Anwendungen frei zur Verfügung. Quelle: DLR 


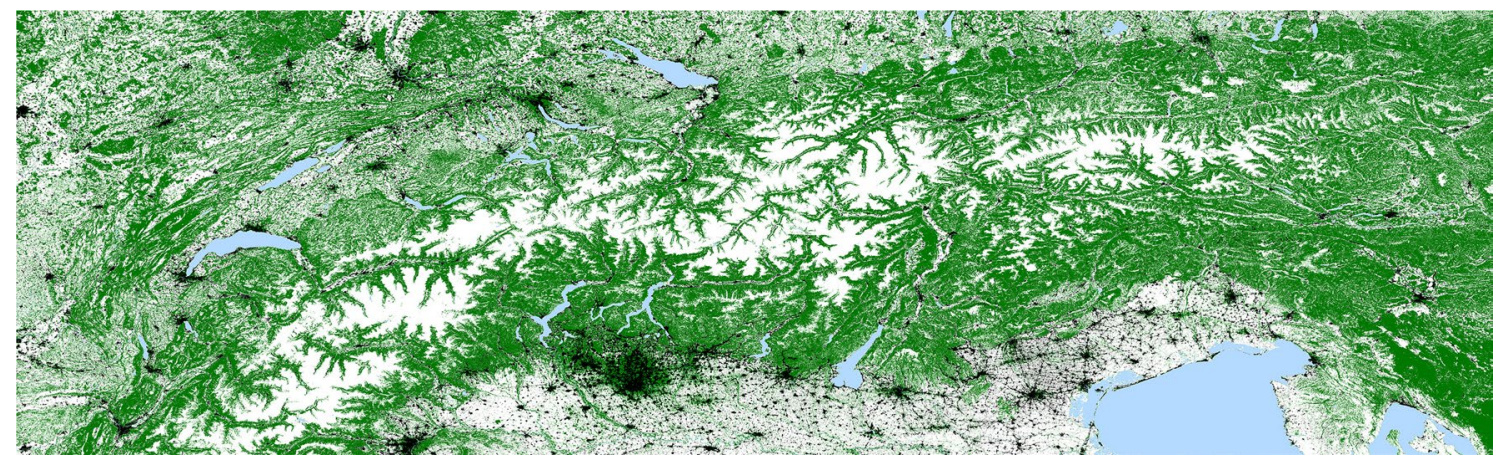

Ausschnitt der TanDEM-X Waldkarte über den Alpen. Quelle: DLR
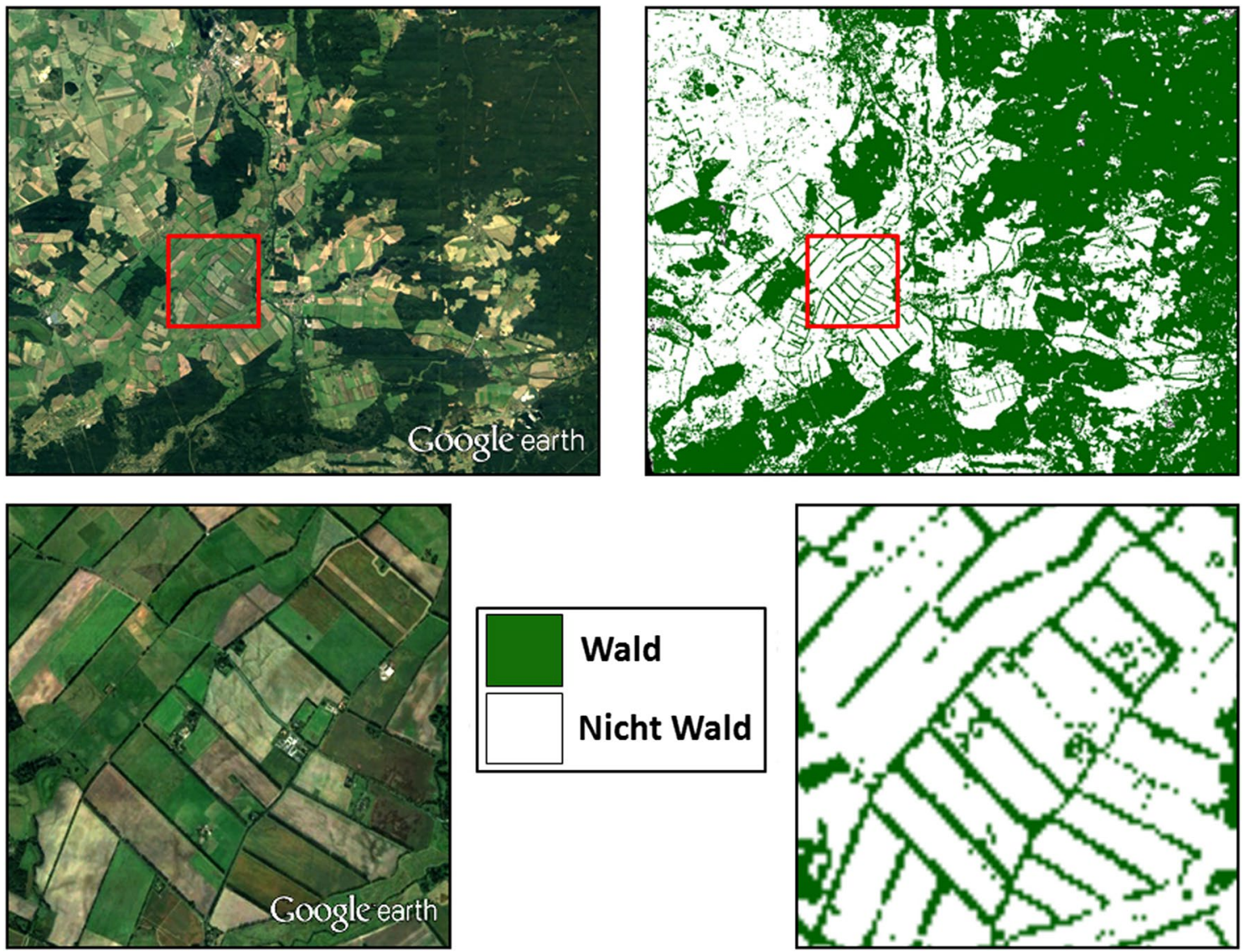

Vergleich einer Google Earth-Aufnahme mit der TanDEM-X Waldkarte für ein Gebiet in Süddeutschland. Im Ausschnitt sind einzelne Baumreihen an Feldgrenzen deutlich erkennbar und demonstrieren so den Detailgrad der Waldkarte. Quelle: Google Earth/DLR

Raumfahrtkontrollzentrum (GSOC) ist für den Betrieb der Radarsatellitenmission TanDEM-X verantwortlich.

Die Abschätzung und das Monitoring von Waldressourcen ist eine zentrale Aufgabe von gegenwärtigen und kommenden Radarsatellitenmissionen. Insbesondere Tandem-L, ein Vorschlag für eine hochinnovative Satellitenmission, könnte globale Waldkarten künftig im Wochentakt erstellen und daraus Waldhöhe, Struktur und Biomasse ableiten. Mithilfe seiner neuartigen Bildgebungstechnologie und der daraus resultierenden gewaltigen Aufnahmekapazität ist Tandem-L dafür konzipiert, weitere dynamische Umweltprozesse auf der Erdoberfläche zu beobachten. Die Mission soll neue Maßstäbe in der Erdbeobachtung setzen und damit einen wirkungsvollen Beitrag zur Bewältigung der globalen gesellschaftlichen Herausforderungen leisten. 


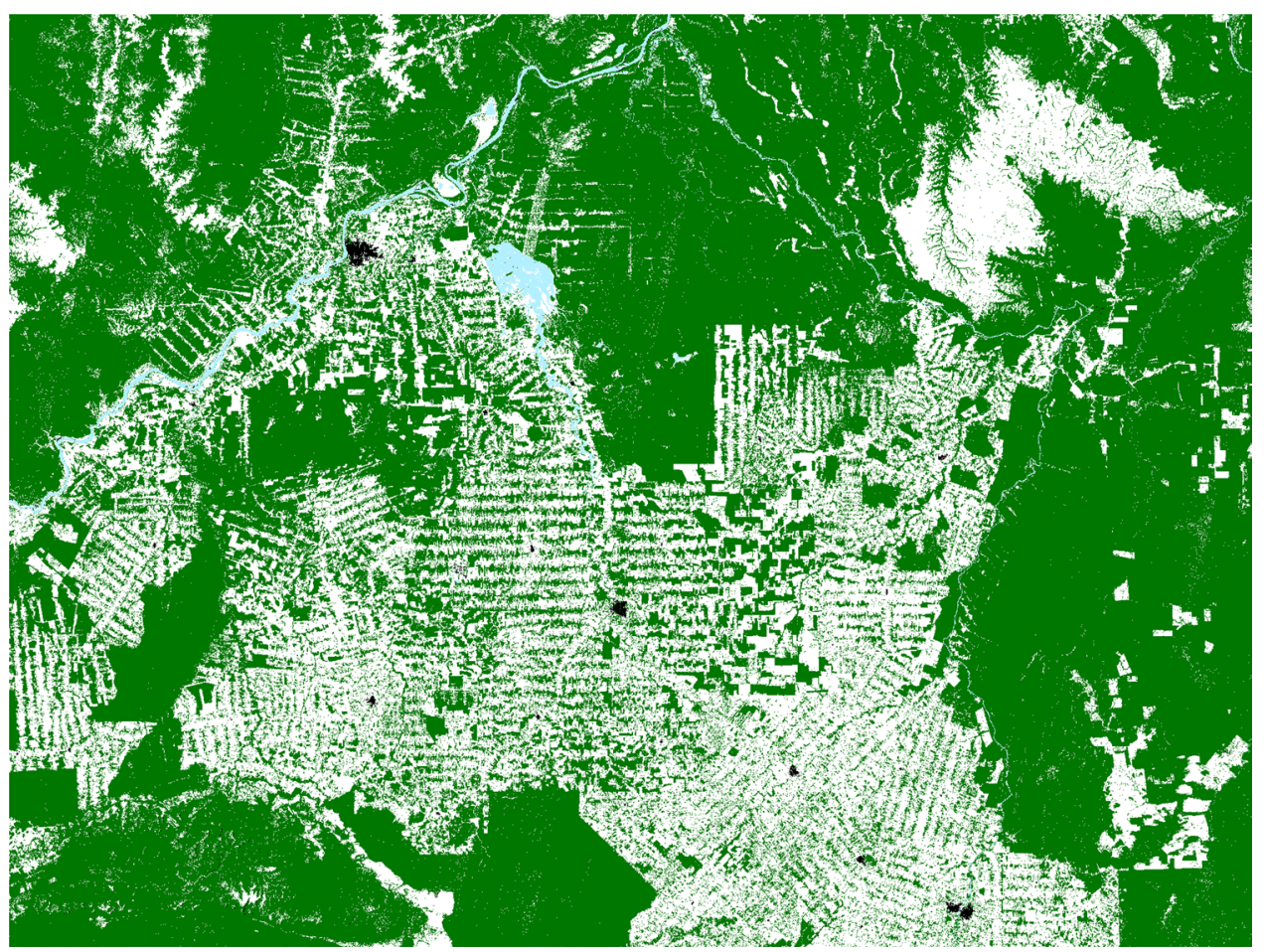

Die TanDEM-X Waldkarte für ein ca. 250 Kilometer x 250 Kilometer großes Gebiet im brasilianischen Bundesstaat Rondonia zeigt die dramatische Entwicklung der Abholzung im Regenwald. Quelle: DLR

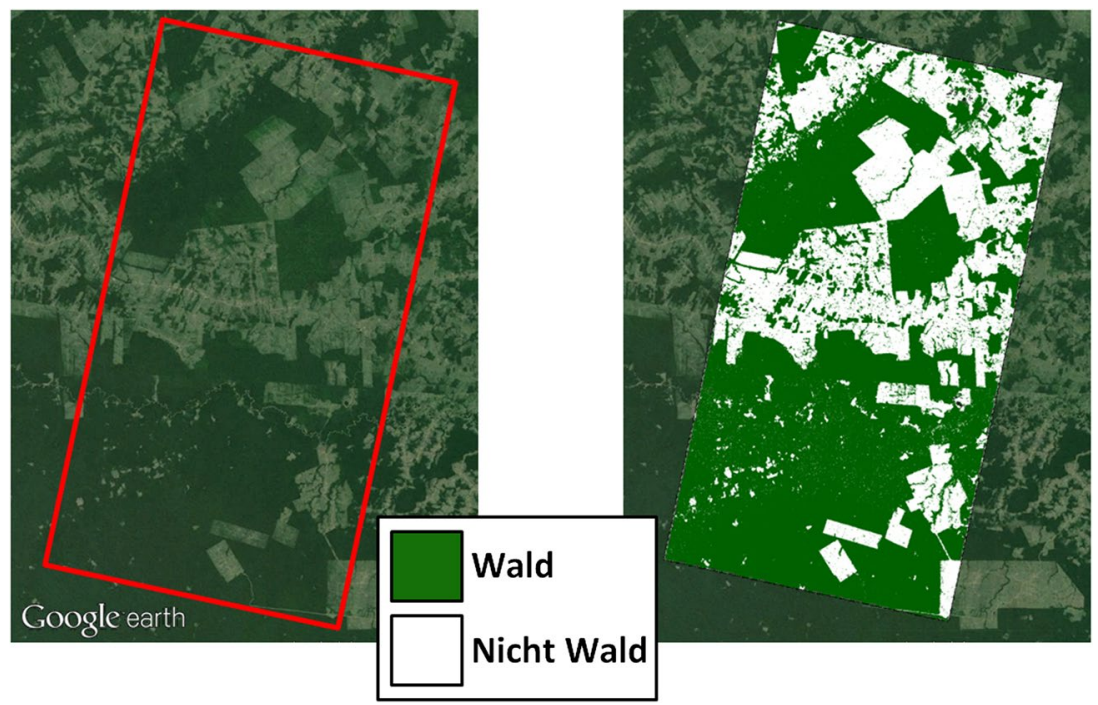

Vergleich einer Google Earth-Aufnahme mit der Waldkarte für ein von Abholzung betroffenes Gebiet im Amazonas Regenwald. Die TanDEM-X-Daten geben erstmals einen genauen Einblick und Überblick über die Abholzungen in den entlegensten Regionen, so dass insbesondere illegale Aktivitäten detektiert werden können. Quelle: GoogleEarth/DLR

- Informationen: Deutsches Zentrum für Luft- und Raumfahrt (DLR); Internet: www.dlr.de

\section{In Heidelberg entsteht ein GeoinformationLab}

In Heidelberg entsteht mit Unterstützung der Klaus-Tschira-Stiftung ein in dieser Form bisher einzigartiges Lern- und Experimentierfeld für raumbezogene Daten, ein öffentlich zugängliches GeoinformationLab.

Das GeoinformationLab soll Forschung, Entwicklung und Ausbildung mit raumbezogenen Daten über die fachlichen Grenzen hinaus ermöglichen.

Raumbezogene Daten (Geodaten) sind Rohstoff der Digitalen Gesellschaft und gleichzeitig auch wesentliche Entscheidungsgrundlagen für effizientes und nachhaltiges Handeln. Dabei sind frei zugängliche Daten sind die Voraussetzung sowohl für eine fundierte Ausbildung in allen Bildungsbereichen (z.B. Schule, Universität, Erwachsenenbildung) als auch die Forschung und Entwicklung neuer datenbasierter Anwendungen und Dienstleistungen.

Zwar haben sich zahlreiche Fachgebiete, z.B. die Geoinformatik oder Data Science, auf die Erfassung, Analyse und Bereitstellung von (Geo-) Daten spezialisiert, es verwundert jedoch, dass Datenlabore als (öffentlich 
zugängliche) virtuelle Lern- und Experimentierräume noch kaum existieren. Das nun startende Projekt zielt darauf $a b$, den Grundstein für ein Geodatenlabor (Geoinformation Lab) mit Fokus auf Geo-, Klima-, Satelliten- und Mobilitätsdaten und dem räumlichen Schwerpunkt der Region Rhein-Neckar zu legen. Damit adressiert das Projekt auch die Bedeutung von Geodaten für die Lösung akuter Herausforderungen im Bereich Mobilität und Klimaschutz der Region. Die Daten und Ergebnisse sollen dabei nicht zwangsweise an der Regionsgrenze Halt machen und grundsätzlich auch Nutzern außerhalb Region zur Verfügung stehen. Erste Ergebnisse sollen sukzessive der Öffentlichkeit zur Verfügung gestellt werden.

Das GeoinformationLab ist ein Netzwerkprojekt des GeoNet.MRN e.V. und offen für alle, die das GeoinformationLab mitgestalten möchten.

- Informationen: Netzwerk Geoinformation der Metropolregion Rhein-Neckar e.V. (GeoNet.MRN), Czernyring 22/11, D-69115 Heidelberg; Internet: www.geonet-mrn.de

\section{ÖKK-Nachrichten / ÖKK News}

\section{Das Jahr 2018 - Aktivitäten in Österreich}

Dieser Bericht schließt an unseren Beitrag in Heft 2/2018 der KN an.

\section{Entwicklungen innerhalb der ÖKK}

Die Mitgliederzahl der ÖKK blieb im Jahr 2018 konstant und beträgt derzeit 124 Mitglieder. Wie üblich fanden zwei Gesamtsitzungen statt: am 11. Juni 2018 im Rahmen des ersten China-Sat Workshops an der Universität Wien und am 15. November 2018 bei der Niederösterreichischen Gemeinde-Datenservice GesmbH, Korneuburg, zum Rahmenthema „Der Einsatz von Geoinformationswerkzeugen im kommunalen Bereich“.

\section{Personalia}

In der Abteilung Kartographie des Bundesamtes für Eich- und Vermessungswesen wurde DI Andreas Pammer ab 01.01.2019 zum neuen Leiter bestellt.
An der Technischen Universität Wien ist die Forschungsgruppe Kartographie ein Teil des Department für Geodäsie und Geoinformation. Zurzeit besteht die Gruppe aus Prof. Georg Gartner und den Forschern Florian Ledermann, Silvia Klettner, Francisco Porras, Wangshu Wang und Andrea Binn.

Prof. Georg Gartner und die TU Wien wurden 2018 Mitglied des Academic Networks of the United Nations Committee on Experts of Global Geospatial Information Management (UN-GGIM).

Seit Februar 2018 ist Peter Jordan Korrespondierendes Mitglied des Ständigen Ausschusses für Geographische Namen (StAGN) (bis dahin: Ständiges Mitglied)

Seit 1. November 2018 ist Benedikt Hajek als Universitätsassistent (prae-doc) in der Arbeitsgruppe Kartographie und Geoinformation am Institut für Geographie und Regionalforschung der Universität Wien tätig.

\section{Kartographiebezogene Veranstaltungen im Inland}

23.04.2018: Amy Griffin, Royal Melbourne Institute of Technology: The user is the centre of the universe: Designing interactive maps for end users (CartoTalks, TU Wien)

09.05.2018: Menno-Jan Kraak, University of Twente: Temporal aspects of O-D matrix visualizations; Lynn Usery, U.S. Geological Survey: A Semantic Approach to Cartography (CartoTalks, TU Wien)

14.05.2018: Evangelos Livieratos, Aristotle University of Thessaloniki: Digitisation and comparison of old and modern maps; Nico van de Weghe, Ghent University: Enrichment, mapping and querying of European traces (CartoTalks, TU Wien)

31.05. - 03.06.2018: Jahrestagung der ARGE Vergleichende Hochgebirgsforschung, Altenberg a. d. Rax

06.06.2018: Sara Fabrikant, University of Zurich: Responsive Geographic 
Information Displays to Support Mobility in the Digital Society (CartoTalks, TU Wien)

11.06. - 12.06.2018: Workshop on Earth Observation with Chinese Satellite Systems and 44th Meeting of the Austrian Cartographic Commission (Universität Wien). Weitere Informationen auf der Webseite: https://china-sat-workshop. univie.ac.at

16.11.2018: Alisa Pettitt, George Mason University: Visualizing Cultural Narratives: Developing XR Applications for Archaeological Sites (CartoTalks, TU Wien)

22.11. - 23.11.2018: Das Internationale Symposion „Minority Names in Public Space - Problems, Challenges, Solutions" fand an der Österreichischen Akademie der Wissenschaften, Wien im Rahmen des Projekts „Politik und Poetik von Toponymen, Identität und Ort in mehrsprachigen Gebieten. Eine vergleichende Untersuchung von Kärnten (Österreich) und dem Teschener Gebiet (Tschechien) " statt. Unter der Leitung von Peter Jordan und Přemysl Mácha stellten die Mitglieder der beiden Projektteams die Inhalte und Ziele des Projekts vor und wurden weitere acht Vorträge zu verwandten Themen gehalten.

Im Rahmen des Aufenthaltes des mittlerweile 7. Intakes der International MSc Cartography - Programmes (gemeinsam mit TU München, TU Dresden und Universität Twente) wurden zahlreiche studentische kartographische Projekte im Bereich WebMapping, Multimedia Cartography, LBS, Cartographic Information Systems, Service-orientierte Kartographie und Map Design erarbeitet.

Weitere Informationen auf der Website: https://cartography.tuwien.ac.at/ great-student-projects-summer-2018/

\section{Wissenschaft}

\section{Diplom- und Masterarbeiten}

Universität Wien - Institut für Geographie und Regionalforschung

Biedermann, Katharina: Die Erstellung von Geländereliefs im Wandel der Zeit - Entwicklung, Anwendung und Perspektiven.

Iglseder, Anna: Detection of surface changes using terrestrial laser scanning: A field study on rock instabilities in the Ybbs Valley, Lower Austria.

Krauthausen, Pascal: Exploring Soccer-Related Graffiti by Applying Geospatial Technology, Geographic Information Systems and Statistical Approaches - A Case Study in Krakow, Poland.

Kröhl, Marie-Luise: Semi-automatic segmentation and classification of Ground Penetrating Radar depth slices and their application in a GIS.

Lukesch, Roland: Performanceoptimierung der Geoinformationsverarbeitung mit $\mathrm{R}$.

Meusburger, Martin: Optimierung öffentlicher Verkehrsnetze mittels GIS.

Resch, Reinhard: Die Problematik automatisierten Erstellens globaler real-time Cloud Cover Mosaike aus frei verfügbaren Satellitenbilddaten.

Stepanek, Katrin: Konzeption und Umsetzung einer eATOC - Aerodrome Terrain and Obstacle Chart - ICAO (electronic) für den Flugplatz Salzburg.

Zanaty, Thomas: Greenfield-Analyse im Retail Banking - GIS-gestützte Filialnetzoptimierung am Beispiel der BAWAG P.S.K.

Zussner, Mario: The Anthropogenic Factor of Natural Disasters
- Determination of potential correlations between soil sealing alongside the Danube River and the extent of the 2013 flood event using geographic citizen science and traditional methods for improving official disaster management.

Technische Universität Wien - Department für Geodäsie und Geoinformation

Kmen, Christopher: Use of Pictures from Social Media to Assess the Local Attractivity as an Indicator for Real Estate Value Assessment.

Neugschwendtner, Martina: Die Rückführung von Katastergrenzen: Ist die Dokumentation von Änderungen im Kataster dafür gerüstet?

Schnitzer, Manuel: Unterstützung der menschlichen Selbstlokalisierung.

Smidek, Julian: Analysis of Movement Data Using ArcGIS in the Cloud.

FH Technikum Wien - Degree Program Intelligent Transport Systems

Inamisami, Victor: Effective pattern for optimal electric vehicle charging stations networking in Vienna.

Raoufi, Yassine: GIS for "Home-Work" Trip Optimization.

\section{Projekte}

Österreichische Akademie der Wissenschaften

Politik und Poetik von Toponymen, Identität und Ort in mehrsprachigen Gebieten. Eine vergleichende Untersuchung von Kärnten (Österreich) und dem Teschener Gebiet (Tschechien) (FWF-Projekt I 2366 G23):

Das Projekt ist vom österreichischen Fonds zur Förderung der wissenschaftlichen Forschung (FWF) aus einer großen Zahl von Einreichungen zur Förderung ausgewählt worden. Es 
wurde in den Jahren 2016-2018 von zwei Forschergruppen in Tschechien (Leitung: Přemysl Mácha, Ostrau) und Österreich (Leitung: Peter Jordan) durchgeführt. Die österreichische Forschergruppe besteht aus drei Personen und ist an der Österreichischen Akademie der Wissenschaften in Wien angesiedelt. Im Kern geht es um die Frage, welche Bedeutung es für Angehörige sprachlicher Minderheiten hat, ihre Ortsnamen auf Ortstafeln und in anderer Weise im öffentlichen Raum zu sehen. Es werden dazu die Minderheitensituationen im südlichen Kärnten und in einem Gebiet mit einer polnischen Minderheit in Tschechien verglichen.

Projekt „Unfolding the Woldan Collection: A data model and prototype for Dissemination and visualization of cultural heritage in a spatial-temporal way“, Sammlung Woldan der Österreichischen Akademie der Wissenschaften (Laufzeit Juni 2018 bis Mai 2020, Projektbeteiligte: Andreas Krexhammer MSc als Projektleiter, Mag. Gerhard Holzer, Dr. Petra Svatek)

Projekt „HistoGIS“, Austrian Centre for Digital Humanities (ACDH) der Österreichischen Akademie der Wissenschaften (Laufzeit: Mai 2018 bis April 2020, Projektbeteiligte: Dr. Peter Andorfer, Mag. Matthias Schlögl)

\section{Universität Wien}

Weinrieden in Österreich: Riedenkarten AT ist ein Forschungs- und Entwicklungsprojekt mit dem Ziel die digitale Erfassung, Darstellung und Präsentation aller österreichischen Weinrieden inklusive der für sie relevanten Faktoren im Rahmen eines digitalen Informations- und Präsentationssystems zu konzipieren, kartographisch umzusetzen und der Fachwelt sowie einer interessierten Öffentlichkeit bereitzustellen. Auftraggeber des Projektes ist die Österreich Wein Marketing $\mathrm{GmbH}$ (ÖWM), durchgeführt in enger
Kooperation zwischen der Universität Wien, Institut für Geographie und Regionalforschung sowie dem Technischen Büro für Landschaftsplanung plan+land, Artner \& Tomasits OG.

\section{Technische Universität Wien}

Wichtige Forschungsprojekte umfassten vor allem das Projekt "Indoor Navi", in dem eine nutzerzentrierte Navigationslösung für Bahnhöfe in Österreich erarbeitet wird sowie das Projekt "EURECA", in dem die Möglichkeiten semantische Informationen aus Archiven so zu extrahieren, dass sie einem Location Based Service zuführbar werden, analysiert werden.

\section{Abgeschlossene Projekte}

\section{Technische Universität Wien}

Die kollaborativ erarbeitete aktuelle Forschungsagenda zum Thema Location Based Services wurde erfolgreich abgeschlossen und als open access paper im Journal of Location Based Services veröffentlicht: https://www. tandfonline.com/doi/full/10.1080/174 89725.2018.1508763.

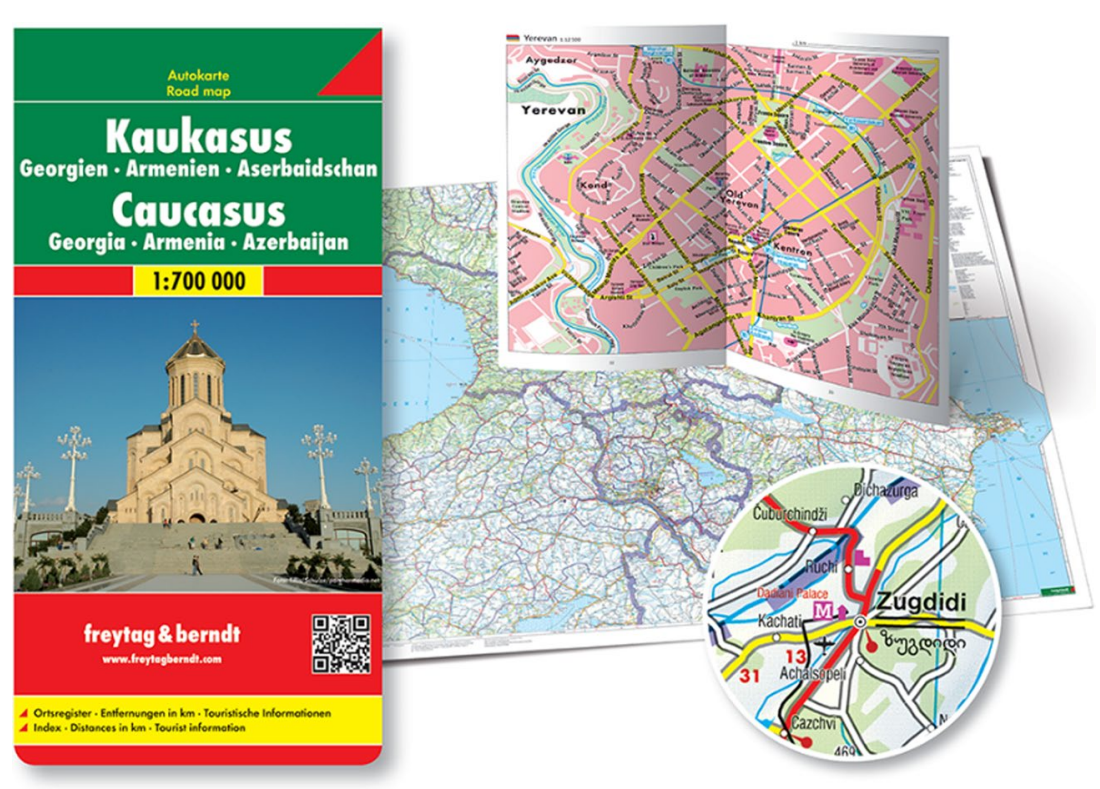

Abb1: Freytag \& Berndt, Autokarte Kaukasus

\section{Amtliche Kartographie}

Bundesamt für Eich- und Vermessungswesen (BEV)

\section{Kartographische Modelle und Kartenwerke}

Für das Kartographische Modell 1:50 000 - Raster (KM50-R) wurde bei 35 Kartenblättern im UTM-Blattschnitt die Flächendeckende Aktualisierung (FAKT) und bei 10 Kartenblättern im UTM-Blattschnitt die Laufende Aktualisierung (LAKT) durchgeführt.

Im Druck sind 33 Kartenblätter der Österreichischen Karte 1:50 000 (ÖK50) und 34 Blätter der Österreichischen Karte 1:25 000V (ÖK25V) erschienen.

Die beiden Kartographischen Modelle 1:250 000 - Vektor (KM250-V) und 1:500 000 - Vektor (KM500-V) wurden mittels der Laufenden Aktualisierung mit relevanten Fortführungsfällen auf den neuesten Stand gebracht und sind als unentgeltliche Produkte über die Homepage des BEV (www.bev.gv.at) verfügbar. 
Digitale Produkte

Die Austrian Map mobile ist eine Softwareanwendung (App) des BEV für das iPhone/iPad von Apple und für Android-Smartphones, mit welcher die kartographischen Daten des BEV auf dem jeweiligen Gerät angezeigt und genutzt werden können. Dieses digitale Produkt des BEV bietet sowohl die hohe Qualität der staatlichen Landkarten Österreichs als auch eine bedienerfreundliche Benutzeroberfläche mit umfangreicher Funktionalität.

Für die Austrian Map mobile als auch die Austrian Map online (www.austrianmap.at) wurden die kartographischen Datenbestände der Maßstäbe 1:50 000, 1:250 000 und 1:500 000 sowie das geographische Namensverzeichnis vierteljährlich aktualisiert.

\section{Internationales}

Das BEV stellte die österreichischen Anteile für die EuroGeographics Produkte EuroBoundaryMap (EBM, 1:100.000) v13.0 und EuroRegionalMap (ERM, 1:250.000) v12.0 bereit.

\section{Lehrlingsausbildung \\ Geoinformationstechniker/in}

Es befanden sich 10 Lehrlinge im 1.-3. Lehrjahr in der Ausbildung zum Geoinformationstechniker/in. Ein Lehrling konnte erfolgreich die Lehrabschlussprüfung ablegen.

\section{Verlagskartographie}

\section{Freytag \& Berndt}

Auto + Freizeitkartenbereich:

Norwegen Atlas 1:250 000/1:400 000

Schweden Atlas 1:250 000/1:400 000

AK0635 Gardasee 1:50 000

AK0211 Thüringen 1:200 000

AK0217 Brandenburg | Berlin 1:200

000

AK154 Kaukasus 1:700 000

AK219 Réunion 1:50 000

AK220 Mauritius 1:50 000
AK215 Kanada 1:3000 000

\section{Stadtplanbereich:}

PL148 Klagenfurt am Wörthersee 1:17 500 mit Softcover

BK100 Wr. Neustadt 1:100 000/1:14 000

Erweiterung der City Pocket Serie wasserfest:

Belgrad 1:10 000

Wander + Freizeitkartenbereich:

Aus der Serie 1:50 000:

WK062 Gesäuse I Ennstaler Alpen | Schoberpass

WK131 Grazer Bergland | Schöckl | Almenland I Stubenbergsee

WK203 Wölzer Tauern I Naturpark I Sölktäler I Rottenmanner Tauern

WK352 Ehrwald I Lermoos I Reutte | Tannheimer Tal | Zugspitze

Aus der Serie 1:35 000:

WK5504 Arlberg I Lech I Sankt Anton I Verwallgruppe

WK5062 Nationalpark Gesäuse । Admont I Eisenerz

Aus der Serie 1:25 000:

WKD4 Garmisch-Partenkirchen I Wettersteingebirge

\section{Verlag Hölzel}

Im Verlag Hölzel ist 2018 ein neuartiger Schulatlas, der ,Hölzel-Aktivatlas" mit einem Atlas- und Übungsteil, erschienen. Zum ersten Mal wird ein Schulatlas direkt auf den Atlasseiten durch Kartenaufgaben erschlossen.

Der Atlasteil enthält einen umfangreichen Topographieblock mit Namenregister und einen nach Schulstufen und Themen des österreichischen Lehrplans der SEK I geordneten thematischen Teil mit kurzen und längeren, einfachen und kniffligeren Aufgaben zur Kartenarbeit auf jeder Seite des Atlasteils.

Im Übungsteil gibt es Arbeitsblätter zur Festigung der Kartenkompetenz in verschiedenen Schwierigkeitsgraden (vom Kartenlesen zur Kartenanalyse).

Nähere Informationen und Probeseiten findet man auf der Verlagshomepage (www.hoelzel.at).
Österreicher im Ausland und bei internationalen Veranstaltungen

Die Forschungsgruppe Kartographie der Technischen Universität Wien war Co-Veranstalter des Workshops "CODE/GEO/GRAPHIC" in Berlin April 19, 2018.

Jordan, P.: Bedeutung und Gebrauch von Exonymen am Beispiel der Slowakei. Universität Hl. Kyrill und Method in Tyrnau, Tyrnau [Trnava], 12. Dezember 2018.

\section{Neuerscheinungen}

\section{Textpublikationen}

Jordan, P., Demhardt, I. \& Losang, E. (Hg.) (2018): Charting the Cosmos of Cartography: History - Names - Atlases (= The Cartographic Journal, 55, 2018, 2). London, The British Cartographic Society - Taylor \& Francis.

Jordan, P. (2018): The Use of Minority Place Names on Maps of the Third Austrian Military Survey - With a Regional Focus on Slovenian and Croatian Lands. In: The Cartographic Journal, 55, 2018, 2, S. 178-186.

Jordan, P., Švehlová, I. \& Woodman, P. (Hg.) (2018): A Survey of Exonym Use. Proceedings of the Working Group on Exonyms Meeting, Prague [Praha], 6-8 April 2017 (= Name \& Place, 7). Hamburg, Verlag Dr. Kovač 2018, 191 S.

Jordan, P. (2018): The endonym/ exonym divide - Questions resolved and still open at the 15 th anniversary of the UNGEGN Working Group on Exonyms. In: JORDAN, Peter; ŠVEHLOVÁ, Irena; WOODMAN, Paul (Hg.): A Survey of Exonym Use. Proceedings of the Working Group on Exonyms Meeting, Prague [Praha], 6-8 April 2017 (= Name \& Place, 7), S. 9-20. Hamburg, Verlag Dr. Kovač 2018. 
Jordan, P. (2018): Exonym use in Austria. In: JORDAN, Peter; ŠVEHLOVÁ, Irena; WOODMAN, Paul (Hg.): A Survey of Exonym Use. Proceedings of the Working Group on Exonyms Meeting, Prague [Praha], 6-8 April 2017 (= Name \& Place, 7), S. 81-94. Hamburg, Verlag Dr. Kovač 2018.

Jordan, P. (2018): Die begrifflliche Unterscheidung zwischen Endonym und Exonym - verdeutlicht am Beispiel von Namen für geographische Objekte im südöstlichen Europa. In: ERNST, Peter; KREVS BIRK, Uršula; PAVIĆ PINTARIĆ, Anita; SCHEURINGER, Hermann; STOJIĆ, Aneta (Hg.): Mehrnamigkeit zwischen Sprachwissenschaft, Sprachgeschichte und Sprachpolitik (= Österreichische Namenforschung Beihefte 6), S. 37-57. Wien, Praesens 2018.

Fischer, K. (2018): „Eine neue Manier, die Stadt Wien und die Leopoldstadt zu befestigen und zu vergrößern." Das Fortifikationsprojekt des Michel Herstal de la Tache in einer Kopie von 1697. In: Elisabeth Loinig, Stefan Eminger, Andreas Weigl (Hg.): Wien und Niederösterreich - eine untrennbare Beziehung? Festschrift für Willibald Rosner zum 65. Geburtstag (St. Pölten 2018) (= Studien und Forschungen aus dem Niederösterreichischen Institut für Landeskunde 70)

Mokre, J. (2018): Über Joseph Daniel von Huber (1730/31-1788) und seine kartographischen Werke unter besonderer Berücksichtigung der perspektivischen Pläne von Prag und Wien. In: Pražský sborník historický 45, Prag 2017, S. 123-172

Mokre, J. (2018): Franz Ritter von Hauslab (1798-1883): A Multifaceted Austrian Representative of the Progress in Cartography in the Nineteenth Century. In: The Cartographic Journal. The World of Mapping 55/2, London 2018, S. 138-149
Mokre, J. (2018): Franz Ritter von Hauslab. Der gelehrte Offizier. Wien 2018 (= Schriften des Heeresgeschichtlichen Museums 25)

Opll, F. \& Scheutz, M. (2018): Die Transformation des Wiener Stadtbildes um 1700. Die Vogelschau des Bernhard Georg Andermüller von 1703 und der Stadtplan des Michel Herstal de la Tache von 1695/97. Wien 2018 (= Mitteilungen des Instituts für Österreichische Geschichtsforschung Ergänzungsband 61)

Kaufmann, V., Seier, G., Sulzer, W., Wecht, M., Liu, Q., Lauk, G. \& Maurer, M. (2018): Rock glacier monitoring using aerial photographs: conventional vs. UAV-based mapping - a comparative study. Int. Arch. Photogramm. Remote Sens. Spatial Inf. Sci., XLII1, 239-246. https://doi.org/10.5194/ isprs-archives-XLII-1-239-2018

Holzer, G. (2018): Jacques Devaulx and Cartography in the Renaissance. In: Élisabeth Hébert, Jean-Yves Sarazin (Hg.), Jacques Devaulx: Nautical Works (2018), 30-69.Svatek, P. (2018): Ethnic cartography and politics in Vienna 1918-1945. In: The British Journal for the History of Science 51/1 (2018), 99-121.

\section{Kartenwerke und Atlanten}

\section{Geologische Bundesanstalt}

Elster, D., Fischer, L., Hann, S., Goldbrunner, J., Schubert, G., Berka, R., Hobiger, G., Legerer, P. \& Philippitsch, R. (2018): Österreichs Mineralund Heilwässer. Wien: Geologische Bundesanstalt, 2018.

Das Hardcover-Buch "Österreichs Mineral- und Heilwässer" ist untergliedert in zwei Teile, die sich zum einen mit Vorkommen \& Hydrogeologie beschäftigen und zum anderen mit Ultraspuren \& Hydrochemie. Beigelegt ist eine Karte von Österreichs
Mineral- und Heilwässern im Maßstab $1: 500000$.

Alpenverein

Alpenvereinskarte 0/16 Mount Kenia Nationalpark inklusive Begleitheft „Das Land um den Mount Kenya“"

In Zusammenarbeit zwischen dem Österreichischen Alpenverein und der Universität Innsbruck erschien im Rahmen des FFG-Projektes AV.MAP die Alpenvereinskarte 0/16 Mount Kenya Nationalpark im Oktober 2018. Ein zentrales Merkmal dieser Karte ist die Nutzung von Satellitendaten, um weltweit ohne Rücksicht auf Landesgrenzen oder Regionen Karten herstellen zu können. Aus hochauflösenden Pleiádes Tri-Stereo Satellitendaten wurden Geländemodelle sowie Orthofotos berechnet. Die zeitliche Übereinstimmung dieser Daten bietet eine gute kartographische Grundlage, aus welcher Höhenlinien, Schummerung und Auswertungen zur Hydrologie generiert wurden. Automatisierte Schutt- und Felsdarstellung ergänzen das Kartenbild. In Verbindung mit weiteren Datenquellen und umfangreicher Geländearbeit ist so eine Trekking-Karte für den Mount Kenya entstanden.

Nähere Informationen findet man auf der Homepage: www.gis.tirol/AV.MAP/

\section{Digitale Werke}

\section{Geologische Bundesanstalt}

IRIS: WebKarte IRIS online - Interaktives RohstoffInformationsSystem

https://www.geologie.ac.at/services/ webapplikationen/iris-interaktives-rohst offinformationssystem/

\section{Sonstiges}

\section{Preise und Verleihungen}

Philipp Gintenstorfer gewann den erstmals verliehenen Förderpreis der ÖKK mit seiner Masterarbeit „Sicheres Verkehrswegemuster? Eine Untersuchung des Einflusses der 


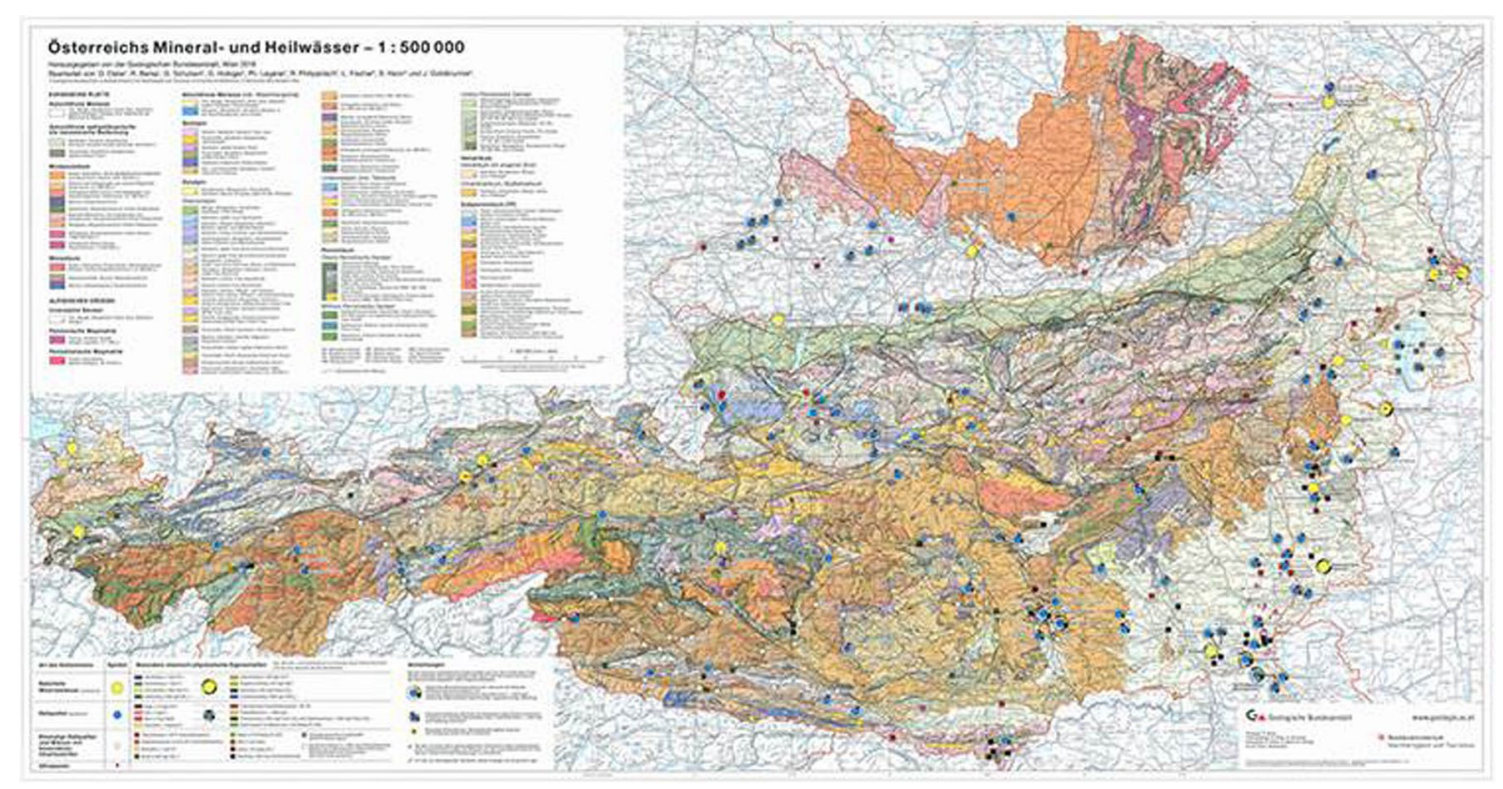

Abb. 2: Geologische Bundesanstalt, Österreichs Mineral- und Heilwasser (orig. Maßstab $1: 500.000$ )

Verkehrsnetzgeometrie auf das Verkehrsunfallrisiko am Beispiel der Stadt Wien".

Florian Ledermann gewann nach dem Best-Teaching-Award der TU Wien (2017), dem 1. Preis bei der Cartographic Exhibition für educational cartographic products bei der ICC 2017 nun auch einen Best-Paper-Award (GI Forum 2018) für seinen Beitrag „Analysing Digital Maps Online: A Reverse Engineering Approach“.

Prof. Georg Gartner wurde am 9.11.2018 von der Eötvös Loránd University in Budapest zum Professor et Doctor honoris causa ernannt.

\section{Arbeitsgemeinschaft für Karto- graphische Ortsnamenkunde (AKO)}

Die AKO hielt im Jahr 2018 ihre 99. und 100. ordentliche Sitzung am 1. März bzw. am 18. Oktober in Wien ab. AKO-Mitglieder nahmen auch an der 143. Arbeitssitzung des StAGN am 26. und 27.02.2018 in Frankfurt am Main, sowie an der 144. Sitzung des StAGN am 12. und 13. November 2018 in Oldenburg teil. Weiters war Österreich durch AKO-Vorsitzenden Gerhard
Rampl und AKO-Mitglied Roman Stani-Fertl auf der UNGEGN-Veranstaltung Scientific Symposium and Joint Divisions and Working Group Meeting vom 10.-14. Oktober in Brüssel vertreten. Das wissenschaftliche Symposium stand unter dem Motto The Role and Importance of Expert Knowledge in the Standardization of Geographical Names. Begleitend fanden zahlreiche Treffen von UNGEGN-Divisionen und Arbeitsgruppen statt. Unter anderem wurde erstmals ein gemeinsames Treffen der Dutch- and German-speaking Division und der Norden Division abgehalten, um gemeinsame Fragestellungen und künftige Kooperationsmöglichkeiten auszuloten.

Die Website der AKO wurde nach dem Wechsel des AKO-Vorsitzes im Jahr 2017 neu gestaltet und ist jetzt unter der Adresse http://ortsnamen. at abrufbar. Neben den AKO-eigenen Inhalten wird auch das Glossary of Terms for the Standardization of Geographical Names für die UNGEGN in allen sechs UN-Sprachen auf dieser Seite zur Verfügung gestellt.

Da die AKO im Jahr 2019 ihr 50-jähriges Jubiläum feiert, wurde von Seiten des Organisationskommitees bestehend aus Peter Jordan, Regina Falkensteiner und Gerhard Rampl mit den Vorbereitungen für eine Festtagung mit dem Thema Names and Migration (vom 6.-8.11.2019 in Wien) begonnen. Weitere Informationen zur Tagung sind auf http://ortsnamen.at/ako50 erhältlich.

\section{Geologische Bundesanstalt (GBA)}

GEOFAST: Das GEOFAST Projekt hat die Erstellung einer flächendeckenden, digitalen Zusammenstellung der jeweils bestverfügbaren geologischen Kartengrundlagen auf Basis des Maßstabes 1:50.000 zum Ziel. GEOFAST-Blätter werden nicht im Offsetdruck hergestellt, sondern als Plot „Print on Demand“ ausgegeben.

Folgende Blätter im Blattschnitt der ÖK50 BMN wurden 2018 erarbeitet:

10 Wildendürnbach, 11 Drasenhhofen, 24 Mistelbach, 25 Poysdorf, 26 Hohenau an der March, 41 Deutsch Wagram, 42 Gänserndorf, 43 Marchegg und 54 Melk.

Benedikt Hajek und Wolfgang Kainz (Vorsitzender der ÖKK) 


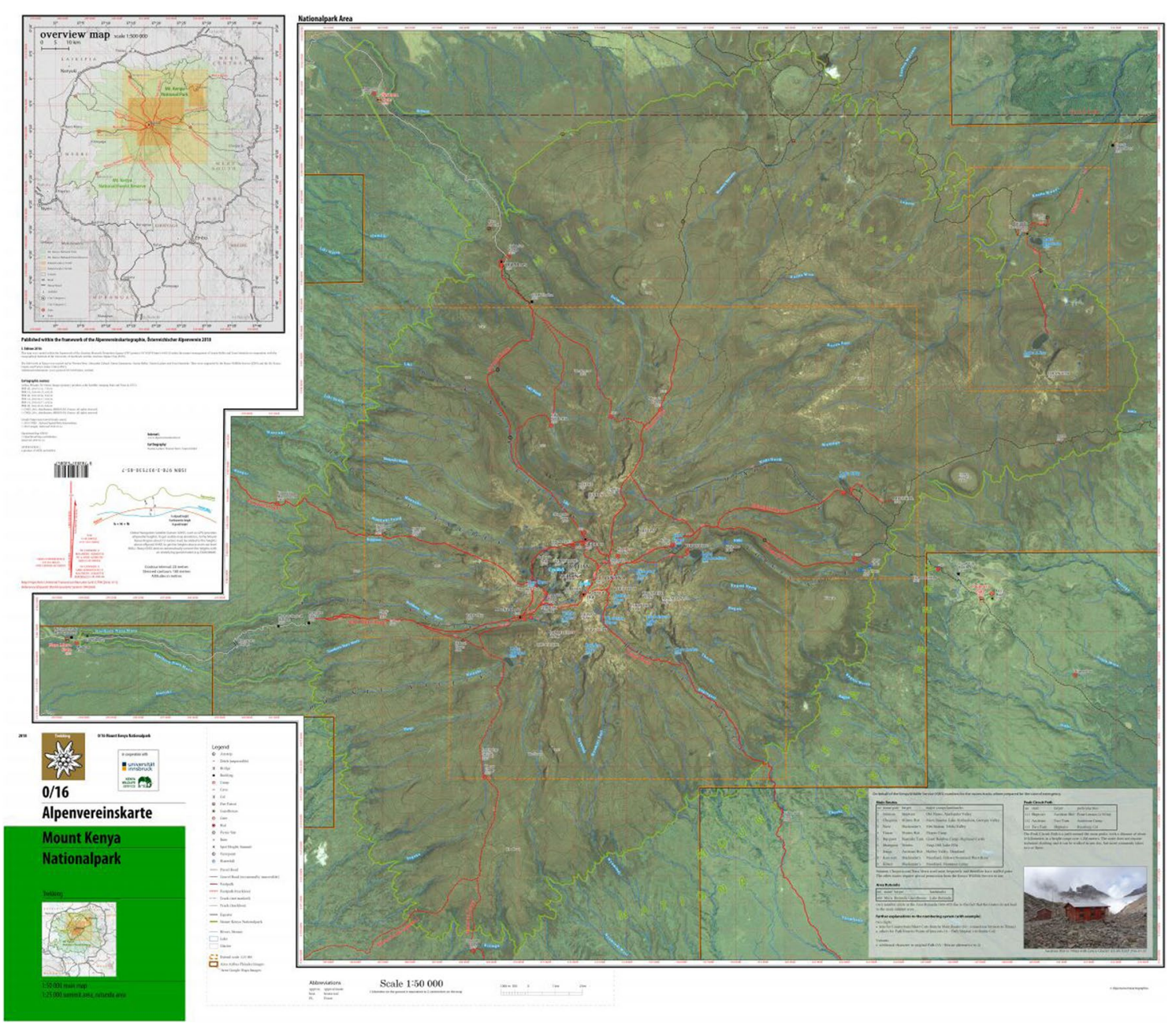

Abb. 3: Alpenvereinskarte Mount Kenia, Vorderseite

\section{Ereignisse/Events}

\section{Deutsches GeoForum}

\section{Call for Papers}

Mit dem 7. Deutschen GeoForum greift der DDGI in seinem 25. Jubiläumsjahr die aktuellen Themen der GeoIT, Erdbeobachtung, Navigation sowie der komplementären Querschnittstechnologien wie BigData, CloudComputing, Digitalisierung, Künstliche Intelligenz und Maschine Learning, auf. Die Geoinformationswirtschaft trägt auf Basis dieser Technologien mit dazu bei, Lösungen zur Bewältigung der großen gesellschaftlichen Herausforderungen der Zukunft zu schaffen: Klimaschutz, Umwelt- und Naturschutz, Ressourceneffizienz und Rohstoffbeschaffung, Erneuerbare Energien, Zugang zu sauberem Wasser, Gesundheit, demografischer Wandel, nachhaltige Landund Forstwirtschaft, Mobilität mit intelligentem, umweltfreundlichem und integriertem Verkehr, sichere Gesellschaften, globale soziale Gerechtigkeit, der demografischen Entwicklung und den Zugang zu Informationen.

Mit der Landesvertretung der Freien und Hansestadt Hamburg als Veranstaltungsort ist zudem ein besonderer thematischer Fokus verbunden. Herausforderungen zur Nutzung von Geoinformationen für Hafenund Küstenstädte sollen dabei u.a. hinsichtlich wirtschaftlicher Aspekte (z.B. Hafenlogistik, Wasserwirtschaft) als auch unter Umwelt- und Sicherheitsgesichtspunkten (z.B. Einfluss des Klimawandels auf Sturmfluten, Starkregenereignisse und Dürre) beleuchtet werden. Die Veranstaltung soll aber nicht auf diese Themen reduziert sein.

Die Geoinformationswirtschaft ist ein starker Motor für die Digitalisierung 


\section{Deutsches GeoForum} Geoinformation für die digitale Zukunft

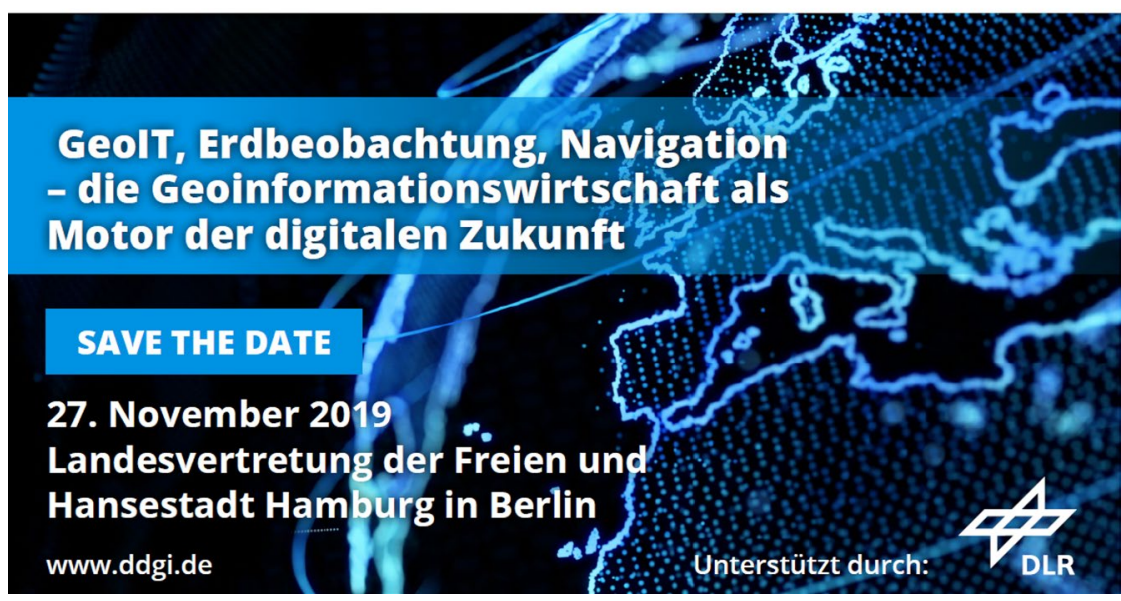

der Zukunft und liefert unter anderem den Treibstoff dafür - die Daten. Erbeobachtungs- und Navigationsdaten von den europäischen Satellitensystemen Copernicus und Galileo, den Geoinformationen aus vielfältigen Sensorsystemen, und Sensornetzen, Fernerkundungsdaten von Flugzeugen und immer mehr auch von unbemannten Luffahrtsystemen - schaffen Beobachtungsdaten in nie dagewesener räumlicher- und zeitlicher Auflösung sowie Datenmengen in neuen Dimensionen von Terabytes und Petabytes - täglich.

Das 7. Deutsche GeoForum bietet Raum für Informationsaustausch zwischen Politik, Wissenschaft, Wirtschaft und Verwaltung.

\section{Die Konferenz}

Datum: 27. November 2019, 9:00 - 16:30 Uhr

Ort: Vertretung der Freien und Hansestadt Hamburg beim BundJägerstraße 1-3, 10117 Berlin

Erwartete Anzahl von Teilnehmenden: 150. Die Teilnahme ist kostenfrei.

Als Key Note ist die Bundesministerin für Umwelt, Naturschutz und nukleare Sicherheit, Frau Svenja Schulze angefragt.

\section{Call for Papers}

Das 7. Deutsche GeoForum soll ein Marktplatz sein und dient dem Wissenstransfer zwischen der Politik, den Technologieexperten, Herstellern und Dienstleistern sowie den Nutzern aus den Anwendungsbranchen und schafft so Impulse für die Entwicklung und Vermarktung neuer Produkte, innovativer Dienstleistungen und intelligenten Lösungen. Gleichermaßen sollen Handlungsempfehlungen für die Politik aufgezeigt werden, die Rahmenbedingungen für die wirtschaftliche Nutzung zu setzen sowie Barrieren und Hemmnisse benannt werden.

Neue Strategien der Open Data Nutzung, auf Anbieterseite wie auf Anwenderseite werden in Zukunft erforderlich sein. Neue IT-Systemarchitekturen, Auswerte- und Analysetools sowie neue Businessmodelle gilt es zu entwickeln.

An den Schnittstellen von Politik, Fachverbänden, Wirtschaft, Verwaltung und Wissenschaft bietet die Konferenz einen aktuellen Überblick zu der wirtschaftlichen Inwertsetzung von Geoinformationen und zeigt die Bedeutung der GeoIT-Wirtschaft für den Wirtschaftsstandort Deutschland auf.
Themengebiete - Technologien - Anwendungen

Sentinels, SAR-Missionen, Optische Missionen (MR, LR, VHR und HR), in-Situ Daten und Systeme, Copernicus-Dienste,...

Themenblock 2: GeoIT - Datenanalyse, Integration, Bereitstellung

mit den Technologie- und Themenbereichen Digitalisierung, Open Data (Gesetzlage national wie EU, PSI-Richtlinie, Zugangsharmonisierung), Big Data (z.B. Veränderung der Geschäftsmodelle - Chancen und Potenziale), CloudComputing, Data Access Points (DIAS, CODE-DE, Hub's...), Künstliche Intelligenz und Maschine Learning, ......

Themenblock 3: Herausforderungen für Anwendungen der Zukunft

$\mathrm{Zu}$ den folgenden drei Themengebieten bitten wir die Vorträge und Präsentationen auszurichten. Bitte geben Sie bei ihrer Einreichung an, welchem Themengebiet Sie Ihren Vortrag und Präsentation zuordnen.

Themenblock 1: Erdbeobachtungsdaten/Copernicus - Sensoren - Dienste

Technische, rechtliche und auch gesellschafltiche Herausforderungen für die zukünftige Nutzung der Geoinformationstechnologien u.a. zu autonomen Systemen - in allen Domänen, Integrierten Anwendungen, Bürgerbeteiligung und Umwelt- und Sicherheitsmonitoring...

Informationen

Die Konferenzsprache ist Deutsch.

Die Präsentationen sollen einen Umfang von 20 Minuten umfassen. Nach jedem Themenblock ist eine Frage- und Diskussionsrunde von 10 Minuten vorgesehen. 
Mit dem „Call for Papers“ möchten wir Sie einladen ein Proposal mit folgenden Informationen einzureichen:

- Titel des Vortrags/der Präsentation

- Name der Autorin/ des Autors/ der Autoren

- Organisation

- Adresse

- Telefonnummer und E-Mailadresse
- Zusammenfassung des Vortrags (ca. $1 / 2$ Seite)

Bitte senden Sie das Proposal an: http://geschaeftsstelle@ddgi.de

Für weitere Informationen stehen Ihnen Herr Hans-Werner Liebers und Herr Peter Loef zur Verfügung, per E-Mail unter http://geschaeftsstelle@ ddgi.de und http://peter.loef@ddgi. deoder Telefon: 0202.4788724 und 0173.5666496.

Einreichungsschluß ist der 27. Juni 2019

Die Auswahl der Präsentationen erfolgt durch den Programmbeirat. Die Ergebnisse des Auswahlverfahrens werden wir bis Mitte Juli 2019 bekannt geben.

Wir freuen uns auf Ihren Beitrag.

\section{DGfK-Nachrichten / DGfK News}

\section{DGfK-Präsident}

\section{Aus der Arbeit des Vorstandes}

Liebe DGfK-Mitglieder,

liebe Leserinnen und Leser,

die Kartographischen Nachrichten

- kurz und griffig die $K N$ - sind das Publikationsmedium der deutschen, österreichischen und schweizerischen Kartographie und Geomatik. Ziel und Bestreben aller, die in der Verantwortung für unser Fachgebiet und unsere Fachgesellschaften stehen, ist, das Ansehen und die Entwicklung dieser Fachzeitschrift positiv zu gestalten und damit Ansehen und Entwicklung von Kartographie und Geomatik im deutschsprachigen Raum zu fördern.

In einem intensiven Diskussionsprozess haben sich alle Verantwortlichen in Funktionen und Gremien der DGfK sowie die Mitglieder eingehend mit der Entwicklung der KN auseinandergesetzt. Dabei wurden die unterschiedlichen Funktionen der KN und ihre Bedeutung in den Bereichen Wissenschaft, Berufspraxis in Wirtschaft und Verwaltung, für Produktinformation und auch als Vereinszeitschrift ebenso gewürdigt wie die sich vor dem Hintergrund dynamischer technischer Entwicklungen genauso dynamisch verändernde Gesellschaft.

Die Sicherung der Qualität und die Weiterentwicklung des Wissenschaftsteils der KN gaben schließlich den Ausschlag für einen Verlagswechsel zur international renommierten Springer International Publishing AG. Dabei wird auch gewährleistet, dass alle weiteren Aufgaben der KN zukünftig weiter erfüllt werden können - ein entsprechendes Engagement aller, die Material für die entsprechenden Rubriken liefern, vorausgesetzt.

Der Vorstand der DGfK - Gesellschaft für Kartographie und Geomatik wünscht dem Hauptschriftleiter der KN, Mark Vetter, mit der gesamten Schriftleitung und dem Editorial Board sowie allen Autorinnen und Autoren einen guten Start in diese neue Epoche unserer Fachzeitschrift. Der $K N$ selbst wünschen wir allezeit qualitativ hochwertige und interessante - sowohl deutsch- als auch englischsprachige Beiträge und die ihr gebührende Verbreitung in den Geo-Fachverbänden in Deutschland, in Österreich und in der Schweiz und nun noch stärker auch in der internationalen Fach-Community

Manfred Weisensee, Hude

Präsident der DGfK - Gesellschaft für Kartographie und Geomatik

\section{DGfK-Mitgliederversammlung 2019}

\section{Einladung}

Hiermit lade ich alle Mitglieder der Deutschen Gesellschaft für Kartographie

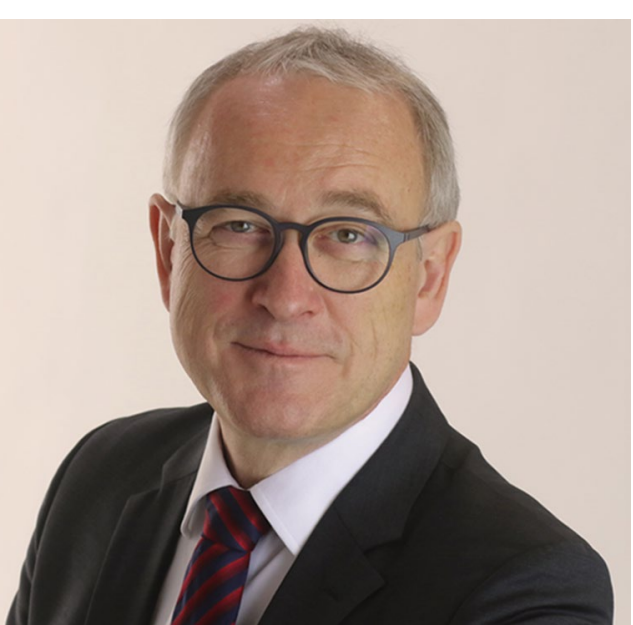

Prof. Dr. Manfred Weisensee

e.V. (DGfK) zur diesjährigen Mitgliederversammlung ein. Sie findet anlässlich des 67. Deutschen Kartographie Kongresses in Stuttgart statt am Montag, dem 16. September 2019, von 14:00 Uhr bis 17:00 Uhr, im Panorama Saal des Landesamtes für Geoinformation und Landentwicklung(LGL), Büchsenstraße 54, 70174 Stuttgart.

Als vorläufige Tagesordnung schlägt der Vorstand vor:

1. Begrüßung und Feststellung der ordnungsgemäßen Einladung und der Beschlussfähigkeit

2. Ehrung der verstorbenen Mitglieder

3. Genehmigung der Tagesordnung

4. Genehmigung des Protokolls der letzten Mitgliederversammlung (veröffentlicht in $\mathrm{KN} \mathrm{3/2018,} \mathrm{S.}$ 165/166) 
5. Ehrung verdienter Mitglieder

6. Bericht des Präsidenten

7. Bericht des Schatzmeisters

8. Bericht des Sekretärs

9. Bericht des Hauptschriftleiters der $\mathrm{KN}$

10. Bericht der Kassenprüfer

11. Aussprache zu den Punkten 6-10

12. Antrag auf Entlastung des Vorstandes

13. Wahlen

- Bekanntgabe der Wahlergebnisse zur Vorstandswahl

- des Wahlausschusses

14. Genehmigung des Haushaltsvoranschlages 2020

15. Künftige Kartographie Kongresse

16. Berichte aus den Sektionen und Kommissionen

17. Verschiedenes

Stellungnahmen oder weitere Vorschläge zur Tagesordnung richten Sie bitte bis zum 6. September 2019 an den Sekretär (http://www.sekretaer@dgfk. net).

Prof. Dr. Manfred Weisensee

Präsident der DGfK

\section{Vorstandswahl DGfK 2019}

\section{Wahlinformation}

Nach $§ 10$ (3) der Satzung der Deutschen Gesellschaft für Kartographie und der Wahlordnung vom 18 . Oktober 2002 sind die Ämter im Vorstand der Deutschen Gesellschaft für Kartographie neu zu wählen:
Präsident/in, Vizepräsident/in (national)

Unter "www.dgfk.net/wahl" finden Sie alle weiteren Informationen zu den Kandidatinnen und Kandidaten und zum Prozedere der Wahl. Alternativ können Sie die Wahlunterlagen postalisch beim Wahlausschuss der DGfK beantragen: Deutschen Gesellschaft für Kartographie e.V. (DGfK), Wahlausschuss, Postfach 11 14, 01686 Weinböhla

Der Vorstand der DGfK

\section{Neue Kommission der DGfK - Virtual und Augmented Reality}

\section{Gemeinsame Arbeitsgruppe der DGfK/DGPF - Virtual und Augmented Reality}

Die Vorstände der DGfK und der DGPF haben im Januar und März 2019 beschlossen, eine neue gemeinsame Kommission zum Thema „Virtual Reality und Augmented Reality“ einzusetzen, um dieses zukunftsträchtige Themengebiet in beiden Gesellschaften zu verankern und zu bearbeiten. Dieses gemeinsame Kommissionsthema verbindet ebenfalls geowissenschaftliche Disziplinen, wie z.B. Geodäsie, Kartographie, GI Science und Geografie, und stärkt die interdisziplinäre Zusammenarbeit mit Nachbardisziplinen, wie z.B. Game Design, Informatik und (Raum-) Kognitionsforschung.

Die Virtual Reality (VR) stellt heute aufgrund der rasanten technologischen
Entwicklung ein wichtiges Werkzeug für viele Anwendungen in Wissenschaft, Industrie und Bildung dar. Durch die Verfügbarkeit von kostengünstigen, qualitativ hochwertigen Head-Mounted-Displays kann der Anwender in eine computer-generierte 3D-Umgebung eintauchen und sich durch diese virtuelle Welt navigieren, um z.B. durch seriöse Spiele oder durch kollaborative Interaktionen die 3D-Welt immersiv zu erleben und zu erforschen.

Augmented Reality (AR) ist die computergestützte Erweiterung der Wahrnehmung von Realität durch die Kombination von Realität und Virtualität. Augmented Reality wird jedoch oft nur als visuelle Repräsentation von Informationen verstanden, d.h. das Hinzufügen von computer-generierten Ergänzungsinformationen oder virtuellen Objekten zu Bildern oder Videos durch Überlagerung in einer entsprechenden Brille, wie z.B. die HoloLens. Entscheidend ist die Überlagerung der Realität durch virtuelle / holographische Inhalte in Echtzeit mit einer kontinuierlichen Anpassung an die Sichtweise des Nutzers. Augmented Reality wird in praktisch allen Bereichen des täglichen Lebens eingesetzt.V.l.n.r.: Virtual Reality Applikation, Navigation in VR, Animation in VR, VR-Brille und AR-Brille im Einsatz

Mit der Leitung dieser gemeinsamen Kommission wurden Prof. Dr.-Ing. Thomas P. Kersten (HafenCity Universität Hamburg) für die DGPF und Dr. Dennis Edler (Ruhr-Universität Bo-chum) für die DGfK betraut. Folgende Arbeitsgebiete (Terms of

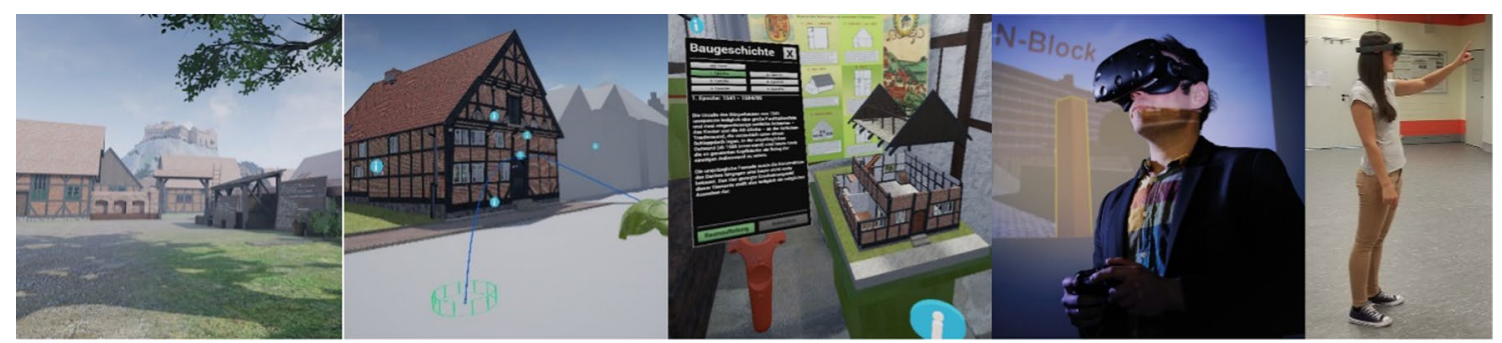

V.1.n.r.: Virtual Reality Applikation, Navigation in VR, Animation in VR, VR-Brille und AR-Brille im Einsatz 
Reference) sind für die nächsten Jahre vorgesehen:

- Innovative VR/AR-Applikationen, Fallstudien

- VR-/AR-Applikationen in der Bildung

- AR/VR-Entwicklungsrichtlinien und Standards

- Mensch-Computer Interface-Design, AR-Benutzeroberflächen

- Innovative VR/AR-Geräte, Methoden und Algorithmen

- Game Engines

- Serious Games (Gamification) und kollaborative Interaktionen

- Bewertung von VR-Applikationen und Leistungsvergleiche

- Anwender-orientierte und kognitionsgestützte Visualisierung von VR-/AR-Modellen

- Einbindung (freier) Geodaten in VR-Landschaften
- Entwicklung und Integration von multimedialen Animationstechniken für die Navigation in AR und VR

- Potenziale von AR und VR für die Visualisierung geographischer und historischer Sachverhalte

- Audiovisuelle Präsentation von VR-Landschaften

- Einsatz von AR und VR in der schulischen und akademischen Lehre

Folgende Tätigkeiten sind für die nächsten Jahre geplant:

- Promotion von VR/AR in der DGPF und der DGfK

- Aufbau eines Netzwerkes von Wissenschaftlern/innen und interessierten Anwendern/innen in VR/AR
- Aufruf und Werbung um Mitgliedschaft in der gemeinsamen Kommission (eMail an Thomas.Kersten@ hcu-hamburg.de oder an Dennis. Edler@ruhr-uni-bochum.de)

- Beiträge für die Sitzungen (Sessions) der Jahrestagungen der DGPF und der DGfK (DKK)

- Jährliche oder zwei-jährliche Workshops über VR/AR in Hamburg, Bochum oder Königslutter

- Veröffentlichung eines Sonderheftes (Special Issue) über VR/AR in der PFG 6/2019 (siehe auch Call for Paper in Heft X/2019)

Informationen über den Arbeitskreis bzw. die gemeinsame Kommission können auch auf der Internetseite abgerufen werden: https://www.dgpf.de/aks/var. html. 


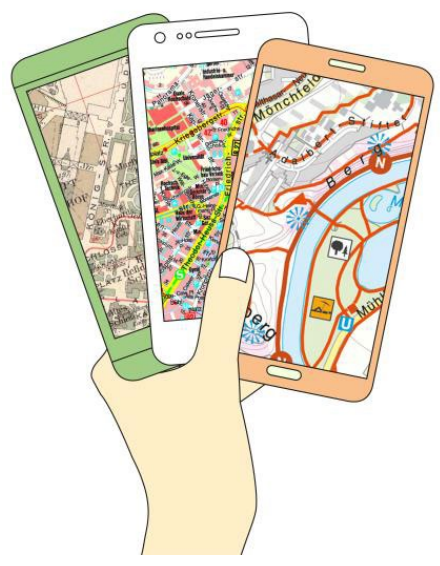

\title{
67. Deutscher Kartographie Kongress 2019 | Stuttgart
}

\author{
17. bis 19. September 2019 \\ im Rahmen der INTERGEO ${ }^{\circledR}$ \\ Ausschnitt aus dem \\ Tagungs-Programm \\ Stand: 24.04 .2019
}

\section{Montag, 16.09.2019}

14:00 Uhr bis 17:00 Uhr

Mitgliederversammlung DGfK

inkl. Vorstandswahlen - siehe gesonderten Aufruf Ort: Panoramasaal im Landesamt für Geoinformation und Landentwicklung Baden-Württemberg, Büchsenstraße 54, 70174 Stuttgart

\section{Dienstag, 17.09.2019}

\section{9:30 Uhr bis 10:30 Uhr}

Eröffnung Intergeo und DKK 2020 \& Keynotes

Smart Cities (Arbeitstitel)

Markus Kerber (Bundesministeriums des Innern, für Bau und Heimat)

Wunderbare Chancen durch digitale Geschäftsmodelle Jürgen Dold (Hexagon Geosystems)

\section{1:00 Uhr bis 12:30 Uhr \\ Innovative kartographische Methoden \\ Leitung: Jochen Schiewe}

Erläuterungen zum Messe-Exponat

Raimar Heber (dpa infografik, Berlin)

Ein Ansatz zur Erstellung von Legenden für Karten mit mehreren Maßstäben

Mathias Gröbe (TU Dresden)

Bewertung optimaler Zoom-Level für die Selbstlokalisation

Bonan Wei; Jochen Schiewe (HafenCity Universität Hamburg)

Denken, Fühlen, Handeln - Eine Taxonomie zur Kategorisierung menschlicher Reaktionen in ortsbasierten sozialen Medien

Eva Hauthal; Dirk Burghardt (TU Dresden)

\section{4:00 Uhr bis 14:30 Uhr \\ Keynote \\ Leitung: Manfred Weisensee}

Wissenschaftskommunikation auf neuen Wegen Hashem Al-Ghaili (Director of Content, Futurism)

Bundeskanzlerin Angela Merkel folgen 2,5 Millionen Menschen, Hashem Al-Ghaili folgen zurzeit 32 Millionen mit steigender Tendenz: Auf Facebook ist der in Jemen geborene und in Berlin lebende Absolvent der Bremer Jacobs University ein Star. In seinen Videos erklärt er anschaulich und unterhaltsam die Welt der Wissenschaft und erzählt von neuesten wissenschaftlichen Durchbrüchen und technischen Errungenschaften.

16:15 Uhr bis 17:30 Uhr

Neue Kommission: Virtual Reality/Augmented Reality Leitung: Dennis Edler

Erläuterungen zur neuen Kommission

Dennis Edler (Ruhr Universität Bochum) \& Thomas

Kersten (HafenCity Universität Hamburg)

Potential eines mittelalterlichen VR-Stadtmodells für den Geschichtsunterricht

Mark Vetter (Hochschule Würzburg-Schweinfurt)

Karlsruher Zeitmaschine - eine VR-App zum Mitnehmen Detlef Günther-Diringer (Hochschule Karlsruhe)

Landschaftstransformation im Ruhrgebiet - Potenziale von Virtual Reality (VR) zur immersiven und multimedialen $3^{D}$-Visualisierung

Dennis Edler; Julia Keil; Frank Dickmann (Ruhr Universität Bochum) 


\section{Mittwoch, 18.09.2019}

9:30 Uhr bis 10:30 Uhr Keynotes

The digitally advanced city: Trusted information whenever and wherever needed

Robert Mankowski (Bentley, Digital Cities Business Unit)

Digitalisierungsstrategie der Landesregierung BadenWürttemberg

Stefan Krebs (ClO Baden-Württemberg)

14:00 Uhr bis 15:30 Uhr

Offene Geodaten in Lehre und Forschung - Praxis-

Workshop zur Plattform OpenEduGeo

Leitung: Ralf Bill

16:00 Uhr bis 17:30 Uhr

Nutzung und Visualisierung amtlicher Daten

Leitung: Andreas Illert

Projekt Smart Villages - Attraktive Orte im Ländlichen

Raum

Gerald Graf (LGL Stuttgart)

Wie produzieren die Schweizer ihre neue

Landeskartenserie?

Mark Wigley (ESRI Schweiz AG)

Aktuelle kartographische Visualisierungen von öffentlichen Statistiken am Bundesamt für Statistik (Schweiz) - Stand und Herausforderungen

Stefan Wondrak_(Bundesamt für Statistik Schweiz)

\section{Donnerstag, 19.09.2019}

\section{9:30 Uhr bis 10:30 Uhr} Keynotes

Smart City: Hype, Utopie, Dystopie - wo stehen wir und wollen wir hin?

Joachim Schonowski (Smart City Experts)

Al und Smart Cities (Arbeitstitel)

Eric Piccuezzu (Autodesk)

11:00 Uhr bis 12:30 Uhr

Anwendungen von Geoinformatik und Kartographie Leitung: Detlef Günter-Diringer

Moornutzung im Verlauf der Jahrhunderte Sandra Schenk; Ralf Bill (Universität Rostock)

Kleinräumige Bevölkerungsdaten und -prognosen im Raster für die Praxis interkommunaler Kooperation Sebastian Specht (OFFIS, Oldenburg)

Mit Karten spielen: Interaktive Kartenanwendungen zum Reflektieren über Fremdsein

Gertrud Schaab, Gertrud; Christian Stern; Jan Jedersberger; Agageldi Samedov (Hochschule Karlsruhe)

\section{4:00 Uhr bis 15:30 Uhr \\ Zugang zu Geodaten und Karten \\ Leitung: Mark Vetter}

Open Data für neve kartographische Produkte Hubert Fünfer (AED-SICAD)

Bedarfsorientierte Forschungsumgebung: Die Geodatenberatung und -vermittlung des FID Karten Wolfgang Crom; Cornelia Koch (Staatsbibliothek zu Berlin)

Digitalisierung der Hochschullehre im Fach Geovisualisierung

Helge Olberding; Mark Vetter (Hochschule WürzburgSchweinfurt) 


\section{Veranstaltungskalender}

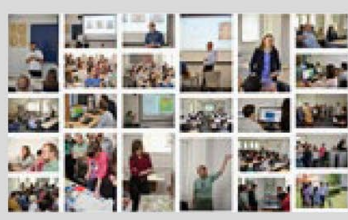

24.06.-30.06.2019

Geospatial Summer School 2019

Palacký University Olomouc,

Department of Geoinformatics

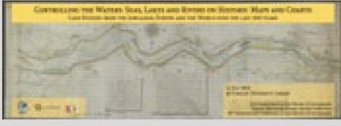

3.07.2019

Controlling the Waters

on Historic Maps

Utrecht University

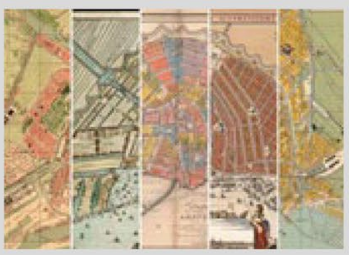

14.07.-19.07.2019

International Conference on the History of Carto-

graphy

Utrecht University

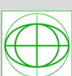

16.09.2019 (11:00-13:30) Vorstandsratssitzung Landesamt für Geoinformation und Landentwicklung (LGL) Panoramasaal, Stuttgart

17.09.-19.09.2019

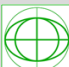

Mitgliederversammlung Landesamt für Geoinformation und Landentwicklung (LGL) -

Panoramasaal, Stuttgart

17.09.-19.09.2019

67. Deutscher Kartographie

Hengress

* Messe Stuttgart 